\title{
Integrins as biomechanical sensors of the microenvironment
}

\author{
Jenny Z. Kechagia ${ }^{1}$, Johanna Ivaska ${ }^{2 *}$ and Pere Roca-Cusachs ${ }^{1,3 *}$ \\ ${ }^{1}$ Institute for Bioengineering of Catalonia, Barcelona Institute of Science and Technology, Barcelona, Spain \\ ${ }^{2}$ Turku Centre for Biotechnology, University of Turku and Åbo Akademi University, Turku, Finland; \\ Department of Biochemistry, University of Turku, Turku, Finland. \\ ${ }^{3}$ University of Barcelona, Barcelona, Spain \\ *e-mail: johanna.ivaska@utu.fi, rocacusachs@ub.edu
}

\begin{abstract}
Integrins, and integrin-mediated adhesions, have long been recognized to provide the main molecular link attaching cells to the extracellular matrix (ECM), and to serve as bidirectional hubs transmitting signals between cells and their environment. However, recent evidence has shown that their combined biochemical and mechanical properties also allow integrins to sense, respond to, and interact with ECM of varying properties with exquisite specificity. Here we review this work first by providing an overview of how integrin function is regulated both from a biochemical and mechanical perspective, affecting integrin cell surface availability, binding properties, activation, or clustering. Then, we address how this biomechanical regulation allows integrins to respond to a broad set of ECM physicochemical properties and signals, such as rigidity, composition, and spatial distribution. Finally, we discuss how this sensing process impacts on major cell functions, by taking cell migration and cancer as examples.
\end{abstract}

\section{Introduction}

By crossing the plasma membrane and linking the extracellular matrix (ECM) to the cell cytoskeleton, integrins are one of the protein families with a more obvious and intuitive structural role. This role as ECMcytoskeletal linkers in fact inspired their name (from their function as "integrators"), and was predicted before the proteins were even identified ${ }^{1-3}$. Since their discovery in the 80 s, integrins have emerged as fundamental cell adhesion receptors, in turn mediating cell and tissue function in a very wide range of scenarios in health and disease $\mathrm{e}^{4,5}$. Integrin function is enabled by a very tight and complex regulation of its properties, both from a biochemical perspective (activation) and a mechanical perspective (mechanotransduction).

Several recent reviews have analysed the details of both biochemical ${ }^{6,7}$ and mechanical ${ }^{8-10}$ integrin regulation, which have been studied extensively. Precisely due to this maturing body of work, it is becoming increasingly possible to elucidate not only how integrins are affected by biochemical and mechanical signals, but also how this sensitivity allows integrins to act as sensors of their environment, the ECM. Due to their binding to ECM proteins, integrins can indeed sense ECM parameters such as its molecular composition and conformation, its physical presentation, its stiffness, or forces transmitted through it. In this review we aim to describe how this sensing occurs. Rather than going into detail about the intricate possibilities of integrin regulation, we will lay out their fundamental biochemical and mechanical principles. Then, we will discuss how those principles enable integrins to sense ECM properties. Finally, we will discuss implications of this process in physiological scenarios, by picking two highly relevant examples: cell migration, and the regulation of dormancy and invasion in cancer.

\section{Biochemical regulation of integrins.}

Before integrins can exert their role as cell adhesion receptors, they need to be transported to the plasma membrane, and activate (that is, change their conformation to enable ECM binding). These steps are directly related to integrin structure, and also provide different means to regulate integrin function. 
Integrins relay signals between the extracellular environment to intracellular response pathways, in both directions ${ }^{11,12}$. These heterodimeric receptors bind different ECM proteins, in a specific manner that derives from at least 24 unique combinations of non-covalently interacting 18 alpha and 8 beta subunits. This facilitates binding to a wide variety of extracellular matrix components as well as counter receptors on other cell types ${ }^{2}$. Although some subunits appear only in a single heterodimer, 12 integrins contain the $\beta 1$ subunit, and five contain the $\alpha \mathrm{V}$ subunit ${ }^{13}$. Both integrin $\alpha$ - and $\beta$-subunits are type I transmembrane proteins composed of a large extracellular domain, single pass transmembrane helices and short cytoplasmic domains (with the exception of the large intracellular domain of the $\beta 4$-subunit). Newly synthesized integrin $\alpha$ - and $\beta$ subunits heterodimerise in the endoplasmic reticulum and are expressed on the cell surface as obligate heterodimers ${ }^{14}$ (Fig. 1). Importantly, the $\beta 1$-subunit is translated in excess, resides in the ER as an immature precursor that matures, and is transported to the plasma membrane only following heterodimerisation ${ }^{7,14,15}$. The integrin ECM-ligand binding sites either comprise epitopes from both subunits (for example in the case of $\alpha 5 \beta 1$ and $\alpha \mathrm{v} \beta 3$ integrins that recognise the RGD-motif in proteins like fibronectin and vitronectin ${ }^{16-18}$ ) or reside on a specific inserted domain of the $\alpha$-subunit (collagen-binding integrins $\alpha 1 \beta 1, \alpha 2 \beta 1, \alpha 10 \beta 1$ and

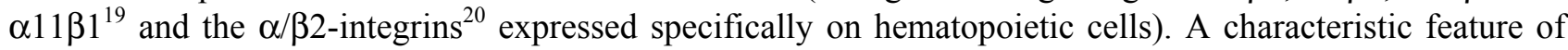
integrins is their ability to bind to several different ECM ligands. ECM ligands, in turn, can also engage different integrin heterodimers ${ }^{13}$. Thus, there is significant redundancy between specific integrins, which has for example complicated the evaluation of the in vivo relevance of these receptors in animal models. However, as discussed here in detail, an emerging theme is that integrins with overlapping ligand specificity possess markedly distinct biomechanical properties. In addition, integrin coupling to other membrane spanning molecules, such as growth factor receptors ${ }^{21}$, proteoglycans ${ }^{22}$ and tetraspanins influence integrin assemblies and their cellular functions. In the specific case of the laminin-binding integrins, their high affinity to tetraspanin CD151 effectively provides them with a "third subunit" that regulates their ECM-binding properties $^{23}$. Thus integrin functions, which have previously been deemed redundant based on shared ligands or joint downstream signalling functions, may emerge as fundamentally distinct biomechanical sensors of the microenvironment.

\section{Regulation of integrin activation by inside-out signalling}

Integrin inside-out signalling (i.e., activation of integrin-ECM binding by cytoplasmic events) has been intensively studied for more than two decades. Originally the focus was on integrins specific for cell types, which, in their unstimulated state, are non-adherent: $\alpha$ II $\beta 3$ in platelets and $\beta 2$-integrin heterodimers in white blood cells. In a resting cell, these integrins adopt a bent inactive conformation but become activated upon stimulation by both agonist stimulation ${ }^{24}$ and force. We will first discuss biochemical signalling pathways inducing integrin activation. The role of force in integrin activation will be discussed in the section "mechanical regulation of integrin conformation/activation" below. The integrin $\beta$-subunit cytoplasmic tail binds to the intracellular adaptor protein talin, which induces separation of the integrin $\alpha$ - and $\beta$-subunit cytoplasmic domains (opening) and triggers a global conformational change in the extracellular domain (which unbends and becomes extended, fig. 1). This leads to a conformational change from a "bent-closed" to an "extended-open" conformation, which has high affinity for ligands, and initiates firm adhesion ${ }^{25}$ (Fig. 1). Talin binding plays a key role in the first steps of integrin activation in all cell types, which is then further supported by the binding of additional cytoplasmic effectors, which mediate not only integrin activation but also the clustering of integrins into many different types of adhesive complexes. Such complexes range from very early nascent adhesions to mature focal adhesions, and are strongly force-sensitive as discussed in more detail below. A major effector is kindlin, which supports integrin-mediated cell spreading via two mechanisms. First, it supports integrin activation through binding to the integrin $\beta$-subunit cytoplasmic tail ${ }^{26}$. Second, kindlin recruits a key focal adhesion component, paxillin, to nascent adhesions to activate Rac, and it directly associates with the actin polymerising Arp $2 / 3$ complex to induce Rac1-mediated membrane protrusions $^{27}$. Upon adhesion maturation, the talin-induced integrin activation can be maintained by tensin 1 and 3 binding to the $\beta 1$-subunit cytoplasmic tail and coupling the integrin to actin ${ }^{28,29}$. Interestingly, the talin 
and tensin binding sites on the $\beta 1$-subunit cytoplasmic tail overlap, indicating that integrins switch from talin binding to tensin binding during adhesion maturation. Tyrosine phosphorylation of the cytoplasmic tail has been suggested to favour integrin-tensin interaction and abrogate talin binding ${ }^{30}$. However, the mechanistic details of the talin-tensin switch in maintaining integrin activity and coupling to actin remain unclear. Conversely, integrin inactivation can be induced by cytoplasmic effectors that compete with talin either directly through binding to overlapping residues on the $\beta$-subunit cytoplasmic tail (these include proteins like $\mathrm{ICAP}^{31}$ and filamin) or indirectly by binding to the integrin $\alpha$-cytoplasmic tail (SHARPIN, MDGI) ${ }^{11}$. Overall, work from adherent cell types such as fibroblasts, epithelial and cancer cells has revealed that spatial regulation of the balance of integrin activating and inactivating proteins modulates cell adhesion, spreading and motility ${ }^{26}$.

A key question related to the first steps of integrin activation has centred on how talin is recruited to the plasma membrane. Single-molecule studies indicated that, unlike integrins that diffuse along the membrane, talin is recruited directly from the cytoplasm ${ }^{32}$ but the mechanism has remained controversial. The prevailing view, largely based on studies in platelets, has been that agonist-induced stimulation of the small GTPase Rap1 recruits the effector protein RIAM that then binds and targets talin to the plasma membrane and integrins ${ }^{33}$. However, an alternative mechanism was recently described where direct interaction between Rap1 and the F0 domain of talin head was suggested to recruit talin to integrins independently of RIAM ${ }^{34}$. This mechanism has previously been dismissed due to the very low affinity of talin-Rap1 interaction in solution. However, in the context of an intact membrane, Rap1-membrane anchoring increases the strength of the interaction, triggering direct membrane targeting of talin by Rap1. Very recently, these data were disputed by a study showing that point mutations within the talin F0 Rap1-binding site have minimal effect on $\alpha$ II $\beta 3$ integrin activation in vitro and in vivo ${ }^{35}$. Therefore, the exact mechanism of talin recruitment to integrins remains to be clarified. Nevertheless, the specific cellular lipid microenvironment is likely to be an important modulator of talin recruitment to integrins. For example, on proteoliposomes, reconstituted $\beta 1$ integrin fragments (membrane-embedded transmembrane-cytoplasmic tail domain) synergized with negatively charged membrane phospholipids (phosphoinositides $\mathrm{PI}(4,5) \mathrm{P}_{2}$ and $\left.\mathrm{PI}(3,4,5) \mathrm{P}_{3}\right)$ to recruit talin head FERM-domain to the membrane ${ }^{35}$. Taken together, these studies indicate that the strength and specificity of integrin proximal protein-protein interactions is strongly influenced by the plasma membrane and this is important to take into account when evaluating the biological relevance of the often weak binary protein-protein interactions of adhesion proteins. Regardless of whether Rap1 recruits talin directly or indirectly via RIAM, this integrin activation step can be inhibited by a family of scaffolding proteins called SHANKs. SHANK1 and SHANK3 share an N-terminal Ras-association (RA) domain with high affinity for Rap1-GTP. By this virtue, SHANKs sequester active Rap1 and limit talin recruitment to the plasma membrane, resulting in reduced integrin activity in cancer cells, primary mammary epithelial cells and hippocampal neurons ${ }^{36}$. Interestingly, at least in the mouse mammary gland, SHANK3 is not expressed in resident fibroblasts. On the same note, in addition to the key role of Rap1, another mechanism of integrin activation has been described in fibroblasts. Here, the central integrin downstream signalling protein, focal adhesion kinase $(\mathrm{FAK})^{37}$, can bind talin directly and recruit it to integrins in newly forming nascent adhesions $^{38}$. Altogether, these important observations suggest that cell-type and/or tissue specific mechanisms may exist to fine-tune integrin activity.

\section{Regulation of integrin cell-surface availability}

Cell-ECM interactions, mediated by integrins, are regulated on multiple levels. As discussed above, the ligand-binding affinity of integrins is under dynamic regulation by inside-out integrin signalling. Furthermore, integrin engagement with ECM ligands and their subsequent clustering triggers accumulation of complex adaptor and signalling protein hubs ${ }^{39}$ regulating integrin downstream outside-in signalling pathways such as activation of FAK, SRC, Akt and ERK pathways and regulation of small GTPases of the Rhofamily ${ }^{5}$. These signalling pathways are essential for many integrin-dependent processes such as cell survival (i.e., prevention of programmed cell death). However, inhibition of FAK or SRC kinase signalling does not trigger gross changes in integrin adhesion complex (IAC) components, indicating that adhesion composition 
is not a reflection of its ability to relay kinase-dependent integrin outside-in signalling ${ }^{39}$. In addition to insideout and outside-in signalling pathways, both active and inactive integrin heterodimers are constantly endocytosed from the cell surface and either continue signalling from endosomes ${ }^{40}$, are recycled back to the plasma membrane to facilitate generation of new adhesion sites ${ }^{41-43}$, or are trafficked for degradation in lysosomes $^{44-48}$ (Fig. 1). Integrins are endocytosed from the cell surface via multiple distinct pathways including clathrin-dependent and independent routes. Clathrin-adaptor proteins can mediate integrin uptake and focal adhesion turnover by binding directly to integrin cytoplasmic tails (e.g. clathrin adaptors Dab2 and ARH to $\beta$-tail motifs ${ }^{49}$; the $\mu 2$ subunit of the AP2 clathrin adaptor complex to a subset of $\alpha$-subunit tails ${ }^{50}$ ). Clathrin-independent integrin uptake is less well understood mechanistically, although it is known that integrin clustering through extracellular lectins induces receptor uptake through the clathrin-independent carrier (CLIC) pathway ${ }^{51}$. The dynamics of integrin traffic play well-established roles in processes such as cell migration and adhesion turnover ${ }^{43,52}$. However, the crosstalk between integrin endosomal traffic and mechanical factors is less clear. While studies directly addressing the effects of cell stretching and plasma membrane tension on integrin endocytosis are largely lacking, membrane tension has strong implications in plasma membrane uptake in general. Increased membrane tension, triggered by osmotic shock or in cells subjected to mechanical stretching, inhibits flat-to-curved transition in clathrin-mediated endocytosis ${ }^{53}$ and also attenuates CLIC fluid-phase endocytosis ${ }^{54}$. Conversely, reduced cell-surface tension induces BARprotein GRAF1-mediated clathrin-independent endocytosis of the plasma membrane ${ }^{55}$. How these relate to regulation of integrin endocytosis or recycling is largely unknown. However, there are some interesting examples of tension/force-dependent regulation of integrin uptake. A study using mobile RGD ligands on supported lipid membranes (RGD-membranes) and rigid RGD ligands on glass (RGD-glass) demonstrated that traction forces inhibit the endocytosis of ligand-bound $\beta 3$-integrins. On RGD-glass, force transmitted from the matrix to integrins blocked Dab2 recruitment to activated $\beta 3$-integrins and the clathrin-mediated myosin II-dependent uptake of these receptors. In contrast on RGD membranes, Dab2 was recruited to integrin adhesions ${ }^{56}$, promoting endocytosis. Interestingly, this contractility dependent clathrin-mediated endocytosis is $\beta 3$-specific and not implicated in the endocytosis of RGD-binding $\alpha 5 \beta 1$-integrin ${ }^{56}$. This is in line with the notion that $\beta 1$-integrin turnover from adhesions is insensitive to force ${ }^{57}$. Interestingly, bone marrow mesenchymal stem cells (BMMSCs) respond to substrate elasticity by regulating the endocytosis and subcellular localisation of collagen-binding $\beta 1$-integrins. On stiff collagen-coated substrates $\beta 1$-integrins are primarily on the plasma membrane, whereas on soft substrates $\beta 1$-integrins are primarily endocytosed via a caveolae/raft-dependent endocytic pathway ${ }^{57}$. In addition, a theoretical analysis based on atomic force microscopy (AFM) data indicated that integrin-ligand complexes are more easily ruptured on soft substrates; this outcome may contribute to the enhancement of integrin internalization on soft substrates ${ }^{58}$. Thus, it is possible that the regulated uptake of different $\beta$-subunit-containing integrins is subject to matrix forces but is mediated by alternative endocytic routes (clathrin-mediated vs clathrin-independent) and may additionally be dictated by other ECM properties, such as matrix constituents; however, these concepts remain to be investigated. Indeed, deciphering the mechanisms bridging mechanobiological signalling to the availability of integrins on the cell surface will be an exciting area of investigation in the future.

\section{The mechanics of integrins.}

Precisely because they connect cells to their microenvironment, integrins are continuously submitted to forces transmitted between cells and the ECM. As such, they are ideally positioned to serve as sensors of mechanical signals. To carry out this function, integrins harness the fact that force applied to macromolecules strongly influences protein conformation and function ${ }^{59}$. In general terms, this mechanical regulation can affect three fundamental integrin properties: i) ligand-binding kinetics, ii) conformation and activation, and iii) clustering and diffusion.

\section{Mechanical regulation of integrin-ECM binding kinetics.}


Perhaps the most obvious effect that force can have at a molecular scale is on the stability of a bond between two molecules. In the simplest case, known as a slip bond, pulling on two molecules forming a bond will tend to dissociate the bond. That is, applied force decreases bond lifetime (or equivalently, increases its off-rate). In physical terms and as first introduced by Bell in the seventies ${ }^{60}$, an applied force will tend to reduce the energy barrier between bound and unbound states, thereby promoting unbinding. However, molecular bonds in general, and integrins in particular, can also exhibit a more counter-intuitive behaviour termed catch bond, or more precisely, catch-slip bond. In a catch-slip bond, applied force first strengthens the bond (catch regime), but once the force surpasses a given threshold it starts weakening the bond (slip regime). Different bond configurations can explain this behaviour ${ }^{61}$, but the most intuitive example is that provided by the analogy of two attached hooks. If no force is applied, the hooks are loosely bound. As one pulls the hooks apart, the hooks first become locked in place, and therefore tightly bound (catch regime). However, if one pulls with sufficient force, the hooks themselves deform and let go (slip regime). Lastly, a final type of bond is that of ideal bonds, in which bond lifetimes do not depend on force. Theoretically, such a regime can be explained by the fact that integrin-ligand bonds involve a complex interaction surface between both proteins, which can be affected by force in more than one way. This can lead to opposing effects of force, that can potentially cancel each other out ${ }^{62}$.

Despite their less intuitive nature, catch bonds seem to be a common feature among integrins (fig. 2a). They occur for instance in different integrin bonds involving RGD-containing ligands, such as those between fibronectin to both $\alpha 5 \beta 1^{63}$, and $\alpha v \beta 3^{64,65}$. Catch bonds have also been widely reported among integrin bonds involved in cell-cell adhesion, such as the bonds between $\alpha \mathrm{L} \beta 2$ and ICAM- $1^{66}, \alpha 4 \beta 1$ and VCAM- $1^{67}$, and $\alpha \mathrm{M} \beta 2$ and ICAM-1 ${ }^{68}$. The specific structural mechanisms underlying integrin catch bond behaviour have been challenging to elucidate, largely because the relatively long times involved in integrin bond dissociation (up to the order of seconds) preclude the use of steered molecular dynamics simulations. In the specific case of $\alpha 5 \beta 1$ integrin, the presence of a secondary "synergy" site for integrin binding in fibronectin (other than the main binding site containing the RGD motif) could also influence bond dynamics ${ }^{69,70}$. More generally, structural data have shown that in extended integrins, the progressive change in orientation from the closed to the open conformation increases affinity for RGD ligands by enabling the formation of hydrogen bonds ${ }^{71}$. Force applied to integrins could thus reduce fluctuations in integrin conformation, locking them in their open state, and prolonging bond lifetimes ${ }^{72,73}$. As we will discuss in the next section, this mechanism exemplifies that any change in integrin conformation will also affect bond response to force, potentially even shifting behaviour from that of catch to that of slip bonds. With respect to the final type of bonds - force-insensitive ideal bonds - they have so far been reported for cadherins ${ }^{74}$, but not integrins. Finally, it is important to note that whereas the properties under force of integrin-RGD bonds have been intensely studied, there are no reports characterizing force-dependent lifetimes of bonds between integrins and important non-RGD ECM ligands such as collagens or laminins. Thus, whether those highly important physiological interactions behave as catch or slip bonds remains an open question, although indirect data suggest some of them could be catch bonds ${ }^{75}$.

\section{Mechanical regulation of integrin conformation/activation.}

Once an integrin is bound to its ligand and force is applied, this is bound to affect not only binding kinetics, but also integrin conformation itself. Since integrins experience major conformational changes during their activation that are essential to their properties, this is very likely a fundamental means to regulate integrin function. Unlike the biochemical "inside-out" activation explained above, this mechanism would operate from the ECM, being classified thus as "outside-in" activation. This hypothesis is supported by molecular dynamics simulations, which have shown that forces can trigger several of the steps involved in integrin activation, essentially by pulling integrins open ${ }^{76-78}$ (Fig. 2c). Indeed and as predicted, experiments pulling on single bonds between fibronectin and integrins $\alpha \mathrm{L} \beta 2^{79}$ and $\alpha \mathrm{v} \beta 3^{64}$ showed that force induces integrins to transition from their bent to their extended configuration. This leads to a drastic change in bond lifetimes under force: ECM bonds with extended integrins exhibit not only overall longer lifetimes, but also a more pronounced catch bond behaviour, which is almost unappreciable 
for the bent configuration. In $T$ cells, force applied to $\alpha \mathrm{L} \beta 2$ integrins by actin flows switches the integrin to an active conformation ${ }^{80,81}$. For $\alpha 5 \beta 1$-integrin bonds, force application also changes integrin activation $^{69}$, even after force is released ${ }^{82}$. This last feature is interesting in that the effects of force persist after application, suggesting a "memory" of applied force encoded in integrin conformation.

Thus, there is feedback between mechanical regulation of integrin conformation and binding dynamics: applied forces will tend to activate integrins and stabilize bonds, and in turn more stable bonds will better resist force and allow it to be transmitted through integrins to further affect their conformation. Additionally, any biochemical interaction affecting integrin conformation/activation (as discussed above) is bound to impact how integrins respond to force. For instance, different conformations of integrin $\alpha 5 \beta 1$ induced by varying ionic conditions can lead the $\alpha 5 \beta 1$-fibronectin bond to behave as either a catch or a slip bond ${ }^{63}$, binding of semaphorin $3 \mathrm{E}$ to the $\alpha 4 \beta 1 / \mathrm{VCAM}-1$ bond drastically reduces lifetimes under force and almost abolishes the catch portion of the catch-slip bond ${ }^{67}$, and binding of ZO- 1 to $\alpha 5$ integrins decreases the lifetime of $\alpha 5 \beta 1$-fibronectin bonds under force ${ }^{83}$. Such tight integration between biochemical and mechanical control of integrin function has recently been proposed to be fundamental and required for the process of integrin activation ${ }^{84}$. In this hypothesis, small forces in the low $\mathrm{pN}$ range would be required to decrease the energy barrier between the bent and extended-open conformations, and enable activation. Such a system could be more sensitive to the activating factor than one in which integrin activation was regulated only by the concentration of cytosolic binding partners such as talin. This is because force would decrease the energy barrier linearly, whereas ligand binding would only do so logarithmically (thereby drastically lowering sensitivity).

\section{Mechanical regulation of integrin clustering.}

Another fundamental integrin property that can be directly affected by force is the clustering of integrins into adhesion complexes, which can occur through different mechanisms. First, once a given cluster of integrins (crosslinked to each other and to actin through adaptor proteins) is submitted to force, it will be subjected to a given elastic strain. It has been hypothesized that incorporating an additional integrin into the cluster will be energetically favourable, simply because overall strain will be distributed among more integrins and thereby relaxed ${ }^{85,86}$ (fig. 2b). Second, the ability of ligand-bound integrins to diffuse laterally may be restricted by the underlying mechanical properties of the substrate, affecting their ability to cluster $^{87}$. Finally, the glycocalyx has also been shown to mechanically promote integrin clustering ${ }^{88,89}$ (fig. $2 \mathrm{~d}$ ). Because the glycocalyx extends from the membrane well beyond the $20 \mathrm{~nm}^{90}$ length of a typical integrin, it serves as a steric barrier impairing integrin-ligand binding. Due to this barrier, once an integrin binds it needs to locally bend the membrane towards the ligand. Subsequent mechanical resistance from the membrane leads to the application of a pulling tensile force on the bound integrin, and a corresponding compressive force on surrounding glycoproteins. Applied force on integrins can then feed back to affect their conformation or binding kinetics. Further, the local membrane deformation induced around the bound integrin acts as a "kinetic trap" where diffusing integrins are closer to the substrate, and thereby have a higher probability to bind ligands. This then promotes integrin clustering, in a way that is also sensitive to the rigidity of the underlying substrate, and ligand density ${ }^{88}$. Other than these three potential mechanisms, mechanical regulation of integrin binding kinetics and conformation can also feed back to affect clustering, as will be discussed in the next section.

\section{Integrins as environmental sensors.}

The exquisite sensitivity of integrins to both biochemical and mechanical signals makes these molecules ideal probes of the cell microenvironment. Interestingly, in most cases combined mechanical and biochemical effects are required to explain cell response. Here we will discuss how integrin properties allow them to respond to three fundamental parameters of the microenvironment: force, rigidity and the presentation and spatial arrangement of the ECM. 


\section{Force.}

Tissues in vivo are continuously subjected to mechanical forces, generated by cells (largely through the contractile action of the actomyosin cytoskeleton), and by indirect factors such as blood flow in endothelia, air flow in respiratory epithelia, or hydrostatic pressure in the mammary gland and bladder ${ }^{91-96}$. Such forces lead to complex tensile and compressive stresses, which cells must sense and respond to in order to maintain homeostasis ${ }^{97}$. Accordingly and because a large fraction of force is transmitted from the ECM to cells through integrins (see box 1), these receptors are considered essential mechanosensors within tissues. Several different single-molecule force sensors have placed the forces experienced by individual integrins within live cells in the wide range of $1-100 \mathrm{pN}^{98-105}$, well within the range where integrin catch bonds (in $\alpha 5 \beta 1$ or $\alpha v \beta 3$ ) have their maximum lifetimes $(20-30 \mathrm{pN})$. Once force is applied to integrins, they respond by the processes of reinforcement and adhesion maturation, which are often used interchangeably but refer to slightly different concepts. Reinforcement describes the increase in the mechanical resistance of integrin-mediated adhesions upon force application. This is usually measured by attaching an ECM-coated probe (such as an AFM cantilever or a microsphere) to cells through integrins, and checking that once force is applied it becomes increasingly difficult to either move the probe or detach it from the cell ${ }^{106-108}$. This initial process can happen within a second of force application ${ }^{109}$, and can likely be explained by the catch bond properties of integrins, without requiring further protein recruitment.

In contrast, adhesion maturation refers to the process by which force application to an integrin-ECM adhesion results in the recruitment of further integrins and adaptor proteins, which link integrins to the cytoskeleton and increase the size of the adhesive complex ${ }^{110-112}$. This process of adhesion maturation is intricate ( see $^{8,113}$ for recent reviews) and can lead to many different types of integrin-ECM adhesive complexes, such as nascent adhesions, focal adhesions, or fibrillar adhesions ${ }^{114}$. However, the general view is that initial, nascent adhesions (with sizes in the order of $100 \mathrm{~nm}$ ) form independently of force ${ }^{115}$, and then mature in response to force application by actin structures ${ }^{65,110,111,116}$, resulting in the alignment between adhesions, actin fibres, and the orientation of forces and integrins themselves ${ }^{117-119}$. This process of course also results in reinforcement of the adhesion, which may be explained partially by direct regulation of integrin clustering as explained above. However, it also involves mechanosensing events at the level of adaptor proteins within adhesive complexes, such as talin, focal adhesion kinase (FAK), or SRC ${ }^{109}$ (fig. 3a). Taking talin as an example, its unfolding is triggered by force ${ }^{120,121}$ and leads to vinculin binding to both talin and actin, strengthening of the adhesion, and eventually adhesion growth ${ }^{65,122}$. However, it is important to note that even if the mechanosensing event does not occur directly in integrins, it is still strongly regulated by the integrin mechanical response. Indeed, forces reach talin through integrins, and the force response of the integrin-ECM bond will determine whether talin experiences forces sufficient for its unfolding. Thus, any event regulating integrin activation or force response (as described above) will also control force response in integrin-mediated adhesive complexes. Because integrin response to force is highly subtype-dependent (as characterized extensively by comparing $\alpha 5 \beta 1$ to $\alpha v \beta 3$ integrins ${ }^{32,57,106,123,124}$, and also $\alpha 5 \beta 1$ to $\alpha 2 \beta 1$ integrins ${ }^{125}$ ) this is also a means to provide molecular specificity to mechanical responses.

An important caveat to consider in this scenario is that, once a given level of force is surpassed, the size of adhesion complexes and the level of force they experience no longer correlate ${ }^{126}$. This shows that adhesions can also grow through force-independent mechanisms, likely mediated by the actin template provided by stress fibres ${ }^{127-129}$. A potential explanation for this is that once adhesions surpass a certain size, force no longer reaches all the parts of the adhesion ${ }^{86}$, leading to a weaker relationship between adhesion size and force. Further, in certain conditions, forces above a given threshold can also disrupt adhesions ${ }^{130}$. In any case, once adhesions form, this then affects cell downstream responses in different ways. Adhesion maturation involves the recruitment and activation of proteins such as FAK ${ }^{131}$, paxillin ${ }^{132}, \operatorname{src}^{133}$, or $\mathrm{ERK}^{112}$. Mature focal adhesions also lead to actin polymerization and the formation of actin stress fibres, which leads to two types of effects. First, actin polymerization directly affects the nuclear localisation and function of transcriptional regulators such as MRTF-A (by releasing it from unpolymerized G-actin ${ }^{134}$ ) or YAP (by releasing it from its binding to the inhibitory SW1/SNF complex ${ }^{135}$ ). Second, stress fibres mechanically 
connect the ECM and integrins to the nucleus via the LINC complex ${ }^{136}$, leading to force transmission from the ECM to the nucleus. This leads to several outcomes, including changes in nuclear pore conformation leading to YAP nuclear import ${ }^{137}$, exposure of specific sites to transcription factors ${ }^{138}$, and changes in the unfolding $^{139}$, accessibility ${ }^{140}$, or phosphorylation ${ }^{141}$ of nuclear proteins (see ${ }^{136}$ for a recent review).

\section{ECM rigidity.}

Another fundamental mechanical property of tissues is their rigidity, and more specifically, that of the ECM (fig. 3b). ECM rigidity results from the combined effect of the composition, degree of crosslinking, and density of ECM components ${ }^{142}$, and it is a major regulator of tissue function. Different tissues and organs within the human body have different levels of rigidity, spanning from very soft tissues in the brain (as low as $10^{1} \mathrm{~Pa}$ in their Young's modulus, a measure of stiffness) to very stiff structures in bone (up to $\left.10^{9} \mathrm{~Pa}\right)^{143}$. Modifications in ECM rigidity are associated with, and drive, several physiological processes, both in healthy scenarios (such as embryonic development ${ }^{144,145}$ ) and in pathological ones (such as cancer ${ }^{146,147}$ ). Whereas forces are actively transmitted through integrins, rigidity is a passive mechanical parameter, which cannot be directly sensed by cells. To probe rigidity, cells need to actively employ their actomyosin cytoskeleton to deform their surrounding ECM through integrin bonds. The resulting forces will depend on ECM rigidity, since a given actomyosin contraction applied by a cell will result in higher or lower forces depending on whether the surrounding matrix is stiff or soft ${ }^{91}$. Thus, cells detect rigidity indirectly by measuring forces, and understanding this process requires dissecting how rigidity regulates force transmission, and how force transmission (as reviewed in the former section) in turn triggers mechanotransduction events in integrins or other proteins.

The processes of both force transmission and subsequent mechanotransduction have largely been studied within the framework of the molecular clutch theory (see box 2 , also recently reviewed in detail ${ }^{91}$ ). In this regard, the properties of integrin bonds (and their regulation by integrin activation and conformation) are a key determinant of force transmission. For instance, we have shown that integrin-fibronectin bonds are only able to sustain the forces required to unfold talin on substrates with rigidities above a given threshold $^{65}$. Thereby, integrin mechanical properties control talin mechanosensing, and set a rigidity threshold for it. Accordingly, cells attaching to fibronectin through different integrins with different mechanical properties ( $\alpha 5 \beta 1$ versus $\alpha v \beta 6)$ exhibit different rigidity thresholds for mechanosensing, and subsequent adhesion formation ${ }^{116}$. Different rigidities can also alter the dynamics of force transmission through the actin-adaptor protein-integrin-ECM "clutch", leading to stable versus spatially fluctuating forces, which then alters cell response ${ }^{132,148}$. Such dynamic force transmission through integrins and associated proteins takes place even at the nanoscale, since actomyosin contractile units at the $100 \mathrm{~nm}$ scale bound to the ECM via integrins have been shown to detect and respond to substrate rigidity ${ }^{149-151}$.

Other than bulk rigidity, whether the ECM behaves as a purely elastic material or has viscous and plastic components (as in physiological ECMs) also strongly determines integrin-mediated responses ${ }^{152,153}$. However, recent work shows that cell response to viscous or viscoelastic environments can also be understood through the clutch theory, simply by considering how the change from elastic to viscous properties affects force transmission ${ }^{154,155}$.

\section{ECM presentation and distribution.}

The specificity of integrins towards distinct ECM ligands implies that different integrins will sense the type of ECM protein they are exposed to. Because some ECM binding motifs are only exposed upon conformational changes (which can be triggered by force) this also implies that integrins can detect the presentation or conformation of specific ECM proteins, and potentially also applied forces through this mechanism. For instance, upon MMP cleavage of triple helical collagen to generate the classic 3/4 and 1/4 fragments ${ }^{156}$ collagen unwinds and can become denatured at physiological temperatures ${ }^{157}$. Partial denaturing of collagen I leads to the exposure of RGD domains, enabling binding to $\alpha 5 \beta 1$ and $\alpha \mathrm{v}$ integrins ${ }^{158}$. Similar 
cryptic domains exist in fibronectin ${ }^{159}$, fibrinogen ${ }^{160}$, and potentially other ECM molecules (see ${ }^{150}$ for a review). However and regardless of conformational changes, another important mechanical (or at least physical) ECM parameter is the spatial distribution of ligands. Of course, parameters such as ECM density will not only regulate rigidity but also ligand availability and spatial distribution. There are several works indicating that ECM ligand density and spatial distribution can affect cell responses per se, independently of rigidity. Specifically, it has been shown by using nanopatterned substrates that integrin clustering and adhesion maturation on stiff environments only occur when integrin binding sites are placed closer than a given threshold, of the order of a few tens of $\mathrm{nm}^{161-164}$. This led to the hypothesis that a putative crosslinking molecule of that size crosslinks integrins to each other, thereby serving as a "molecular ruler" measuring ligand spacing ${ }^{161,165-167}$. However, recent work has shown that this effect of ligand spacing is also mechanical. Indeed, it is straightforward to predict that the transmission of actin contractility to the ECM will result in different forces per integrin if the overall distribution and concentration of available binding sites is altered (Fig. 3c). This has indeed been confirmed ${ }^{168}$ using single-molecule force probes. Consequently, the effect of ECM ligand distribution on integrin forces determines the specific thresholds of both ligand spacing and substrate rigidity that will enable mechanotransduction, and subsequent adhesion maturation ${ }^{130}$.

\section{Roles in physiology and disease.}

As both a structural scaffold and a conveyor of biochemical and mechanical signals, the ECM regulates a very wide range of processes, from single cell events driving cell cycle progression ${ }^{12,}{ }^{13}$ to complex events in cancer or embryonic development. In such in vivo scenarios where processes occur over the long term, it is interesting to note that integrins not only sense the ECM, but are also able to regulate it, thereby setting up feedback systems. For instance, in ventricular fibrosis force transmitted through integrins can lead to secretion of TGF- $\beta$ to the microenvironment, initiating signalling ${ }^{169,170}$. In migration and cancer, such forces can also remodel ECM architecture, as we will discuss below. Whereas covering all such relevant physiological scenarios would not be feasible within the scope of this review, we will discuss two cases exemplifying the importance of integrin-mediated ECM interactions: cell migration within morphogenetic events, and the control of cell dormancy and invasion in cancer.

\section{Cell migration and morphogenesis.}

Cell migration is essential for a wide range of biological processes such as embryo morphogenesis, wound healing, or regeneration. It is also fundamental in pathological conditions such as cancer, where cells migrate either as individual entities or as highly coordinated collectives in response to molecular and mechanical cues from their environment. Directional cell migration is guided by gradients of different environmental cues, such as diffusible ligands (chemotaxis), substrate-bound ligands in the ECM (haptotaxis), or ECM rigidity (durotaxis) ${ }^{171,172}$ (fig. 4a). Integrins play an essential function in haptotaxis and durotaxis, which naturally derives from their capabilities to sense ECM rigidity and distribution as described above. Indeed, integrin-mediated molecular clutch mechanisms have been proposed to explain cell migration speeds in response to ECM rigidity ${ }^{173}$, and directional migration in response to ECM rigidity gradients (durotaxis), in the context of both single cells ${ }^{132}$ and collective migration ${ }^{174}$. In all cases, the integrin response would be regulated (among other parameters) by the type of integrin-ECM bond being formed, integrin activation state, and myosin contractility levels, providing precise molecular and cell-type specificity to the ECM response.

In vivo, there are different examples highlighting the importance of migration. In the developing mammary gland, mammary epithelial cells migrate collectively into the fat pad, and this process is guided by cell-extrinsic stromal cues ${ }^{175}$ (fig. $4 \mathrm{~b}$ ). The major structural protein of the mammary gland is fibrillar type I collagen, which underlies the basement membrane that separates the epithelium from the less structured underlying collagen I-rich stromal compartment ${ }^{176}$. Aligned collagen fibres, deposited and remodelled by the mammary gland stromal macrophages ${ }^{177}$ and fibroblasts ${ }^{178}$, guide the invading epithelial cells giving rise to the nascent ducts which are led by the specific tip structures called terminal end bud 
$(\mathrm{TEB})^{179}$. Collagen alignment in the mammary gland provides topological cues to guide migration. It also correlates with tissue rigidity during development ${ }^{178}$ and during cancer progression ${ }^{180,181}$, suggesting that integrin-dependent rigidity and ECM sensing could be fundamental to the process. Highlighting the involvement of integrins, stromal deletion of the integrin inhibitor SHARPIN gives rise to impaired alignment and reduced tissue stiffness in vivo, possibly switching ECM rigidity away from the stiffness optimum of the collectively migrating mammary epithelial cells.

Another example of rigidity guided tissue morphogenesis is the migration of the Xenopus laevis neural crest cells (fig. 4c). This embryonic cell population undergoes developmental epithelial-to-mesenchymal transition (EMT) and become migratory ${ }^{182}$; a process with many parallels to invasive migration of human carcinomas $^{183}$. In the developing embryo, tissue rigidity increases locally coinciding with the onset of EMT and collective cell migration from the neural crest. Remarkably, rigidity governs the migration response to chemotactic cues from a critical morphogen SDF-1, enabling only cells on a sufficiently rigid fibronectin support to respond. Furthermore, tissue stiffening above the critical threshold is sufficient to trigger premature migration of non-migratory neural crest cells in vivo. In this setting, mechanosensing was reported to be dependent on $\beta 1$-integrin/vinculin/talin-mediated mechanosensing and myosin contractility ${ }^{145}$, suggesting an involvement of the integrin-talin clutch mechanism described above. Thus, mounting evidence suggests that the mechanobiological principles discovered initially in vitro, using model systems, are valid in vivo and play fundamentally important roles during development and cancer.

\section{Tissue rigidity and ECM remodelling in dormancy and invasion.}

The ECM in tissue stroma is composed of a complex meshwork of highly cross-linked proteins. The physical properties and architecture of the ECM are highly tissue specific, ranging from interstitial forms within organs to specialized forms, such as basement membranes underlying epithelia and the vascular endothelium $^{184}$. The chemical composition and the biomechanical properties of the ECM are key to maintenance of tissue homeostasis and altered ECM properties underpin many human pathologies including cancer progression and dissemination ${ }^{185}$. As expected, the fundamental role of integrins as ECM sensors places them as major players in this response. The most abundant ECM proteins are collagen and fibronectin and assembly of a fibronectin network is often the "seed" and prerequisite for deposition of 10ibrillary collagen networks ${ }^{186}$. Fibronectin network assembly requires fibronectin-binding integrins, especially $\alpha 5 \beta 1$, and mechanical stimulation provided by cellular traction forces. Fibronectin has a high degree of conformational flexibility and forces transmitted from $\alpha 5 \beta 1$ integrins to fibronectin expose cryptic binding sites in fibronectin necessary for its polymerization ${ }^{187}$. During cancer progression, the tumour stroma is remodelled in multiple ways resulting in increased tissue stiffness, altered biochemical composition and cancer-specific fibre alignment ${ }^{146,188}$. Activated fibroblasts in the cancer stroma, referred to as cancer associated fibroblasts (CAFs), are the main architects of the cancer stroma through their ability to deposit ECM and to physically remodel the stroma ${ }^{189}$ (fig. $4 \mathrm{~d}$ ). Increased collagen deposition and crosslinking facilitate cancer progression by activating integrin downstream signalling pathways such as FAK and YAP/TAZ. These have been reviewed extensively in several excellent reviews ${ }^{8,190}$ and we will discuss here only some recent advances in how tumour stroma remodelling and CAFs drive invasion and regulate awakening of dormant disseminated cancer cells.

Cancer dissemination requires the ability of cancer cells to break through the basement membrane and navigate away from the primary tumour. In vivo imaging of breast cancer specimens has suggested that CAFs contribute to this by remodelling "tracks" of collagen to facilitate invasion ${ }^{181}$. In addition, the tumour stroma is enriched in secreted fibronectin and tenascin $C$ that favour tumour progression ${ }^{191,192}$. However, more recently, the role of physical remodelling of fibronectin has been highlighted in cancer invasion (fig. 4d). In prostate cancer, CAFs assemble a fibronectin-rich matrix with anisotropic fibre orientation. They remodel fibronectin through actomyosin-contractility driven traction forces and $\alpha 5 \beta 1$ integrin to generate aligned fibres that guide cancer cells to promote directed cell migration ${ }^{193}$. In contrast, in models of colon cancer, CAFs induce invasion of cancer cells through increased fibronectin secretion 
and $\alpha v \beta 3$-driven matrix remodelling ${ }^{194}$. Interestingly, in this system the two fibronectin binding integrin $\alpha v \beta 3$ and $\alpha 5 \beta 1$ played sequential roles and both were required for invasion. In a collagen matrix, with CAF-secreted fibronectin matrix, $\alpha v \beta 3$ clustered first, in line with its role in force-dependent focal adhesion maturation ${ }^{107,115}$. $\alpha 5 \beta 1$ integrin was recruited to fibrillar adhesions later and was critical only at later stages of fibronectin assembly and generation of myosin contractility at these sites ${ }^{194}$. Whereas not directly addressed in these studies, it is interesting to speculate that the differences in force response between the two integrin types could explain their different roles, for instance by triggering talin or FAKmediated signals at different force thresholds. These studies also highlight the fundamental "seeding" role of fibronectin and suggest that it may be a key ECM mechanotransducer in the stroma owing to the effect of force on its conformation.

CAFs are additionally implicated in the switch from carcinoma in situ to an invasive carcinoma. This step involves cancer cells breaking through the underlying basement membrane to the underlying stroma and is generally considered to be regulated by cancer cell secreted proteases such as the collagenase MT1$\mathrm{MMP}^{195}$. However, CAFs may contribute to this process through their unique biophysical properties. A recent study, using decellularized mouse mesenteric basement membrane, demonstrated that CAFs interact with the basement membrane presumably through integrins and pull and stretch the matrix, triggering softening and eventually the formation of gaps that are permissive for cancer cell invasion ${ }^{196}$. Another barrier for tumour dissemination may lie within the mechanical properties of the polarized glandular architecture of the mammary gland epithelium. It is composed of basal myoepithelial cells adhering to the basement membrane and apically positioned luminal cells ${ }^{197}$. Several transcription factors involved in EMT, such as TWIST, are linked to poor prognosis and metastasis in breast cancer ${ }^{198}$. Integrin- $\beta 1$ is upregulated by TWIST in breast cancer and contributes to increased invasion ${ }^{199,200}$, suggesting that integrin-linked EMT-type changes contribute to breast cancer metastasis. Accordingly, expression of TWIST1 in mammary gland myoepithelial cells increases cancer cell dissemination into the surrounding tissue $^{201}$. However, invasion induced by luminal-specific activation of TWIST was mechanically blocked by the underlying genetically normal myoepithelial cells. These were capable of capturing the invading luminal cells forcing them back into the epithelial structure ${ }^{201}$. While the exact role of integrins was not studied here, at least two potential biomechanical mechanisms could be at play here: first, activation of TWIST downstream of integrin-mediated mechanosensing ${ }^{202}$, and second, changes in mechanosensing induced by a switch in integrins with different properties under force, from integrin types dominant before EMT to upregulation of integrin $\beta 1$. In any case, these data indicated that the biomechanics of the tissue have the capacity to act against EMT-driven integrin-linked invasion.

Disseminated cancer cells can remain dormant in tissue over extended periods of time, and the cues triggering awakening are still largely unknown. However, integrin-mediated adhesion is key to several of the mechanisms described thus far. $\alpha 5 \beta 1$ integrin association with the urokinase plasminogen activator receptor (uPAR) can trigger FAK and ERK-dependent escape from dormancy ${ }^{203}$ and integrin-rich filopodia-type adhesions driven by formin-mediated actin polymerization are linked to overcoming dormancy $^{204}$. In dormant breast cancer cells, contacts with fibrotic and stiffer collagen-rich ECM triggers awakening through $\beta 1$-integrin activation of SRC and FAK, leading to induced actomyosin contractility and actin stress fibre formation in the cancer cells ${ }^{205}$. Importantly, the transition from quiescence to proliferation is initiated by fibronectin secretion into the ECM and can be overcome by inhibition of $\beta 1$ integrin or cellular contractility ${ }^{206}$, suggesting that integrin-mediated forces regulate the transition from a quiescent to a proliferative state in vitro. Sustained inflammation can also drive integrin-dependent proliferation of dormant cells. $\beta 1$-integrin-mediated cell adhesion to laminin can suppress proliferation and malignant features on breast cancer cells ${ }^{207}$. However, during sustained lung inflammation, neutrophilreleased proteases process laminin via a two-step processing giving rise to laminin fragments that activate $\alpha 3 \beta 1$-integrin and its downstream signalling to induced proliferation of dormant cells in vitro and in vivo $^{208}$. This was linked to increased cell tension after binding to processed laminin, suggesting that integrin-mediated mechanics are key regulators of disseminated cancer cells in the lung ${ }^{208}$. This is 
concordant with the notion that increased ECM stiffness in lung fibrosis models induces cancer cell awakening from dormancy ${ }^{205}$. Again, the involvement of different ECM and integrin types, and of mechanical factors, strongly suggests that the different properties under force of each integrin-ECM pair may be a fundamental driver of the specificity and context-dependence of dormancy and subsequent awakening.

\section{Conclusions and perspectives}

By combining sensitivity to both biochemical factors and mechanical forces, integrins are poised to exert a fundamental regulatory role not only in the examples described here, but virtually in any tissue remodelling scenario. In this regard, a fundamental challenge is to link specific sensing mechanisms (precisely dissected in simplified single molecule or cell culture systems) to observed effects in vivo. As exemplified in the last section of this review, this link is often unclear, and unravelling it will require the development of engineered setups combining sufficient levels of complexity while retaining the ability to perform precise mechanical and biochemical measurements. This is critical not only to unravel the fundamental science involved, but also to design potentially more precise integrin-based therapies in ECM-related diseases such as cancer or fibrosis. Attempts to target integrins in the treatment of solid tumours have often failed in clinical trials, potentially due to the very general, structural function of integrin adhesion in adherent cells, and their inherent redundancy. Additional complications may have arisen from possible side effects such as promoting angiogenesis $^{209}$, or metastasis by aiding in cancer cell detachment from primary tumours ${ }^{210}$. In contrast, a precise understanding of the combined biochemical/biophysical mechanisms involved in integrin sensing could lead to more focused strategies. For instance, therapies specifically designed not to block adhesion, but to inhibit or modulate integrin-mediated mechanosensing, have not been attempted so far and could open an entirely new approach to the therapy of diseases like cancer. 


\section{Boxes and figure legends}

Box 1: Force transmission through integrins. Because integrins link the ECM to the actin cytoskeleton, they withstand and transmit cell-ECM forces. For instance, any force applied to an ECM fibre will pull on ligand-bound integrins. Whereas integrins do not directly bind actin, their cytoplasmic tails bind to adaptor proteins like talin. Several such integrin adaptor proteins (such as talin, tensin, filamin, or $\alpha$-actinin) bind in turn to actin, and thereby transmit applied forces from integrins to the actin cytoskeleton. Conversely, forces applied to actin (via myosin contraction or actin polymerization) are transmitted to the ECM through adaptor proteins and integrins. This general path of force transmission (ECM-integrins-adaptor proteins-actin) is clear, and adaptor proteins like talin ${ }^{211,212}$ and vinculin ${ }^{213}$ have been measured to be submitted to force. However, how force is distributed among the many adaptor proteins that directly or indirectly link actin to integrins remains unclear. It is also important to note that the ability of integrins to transmit force is highly regulated by their linkage to both ECM and actin. For instance, if an integrin is pulled through an ECM link but the integrin is not linked to actin, the integrin will merely slide along the membrane and not effectively transmit force. The same thing will happen if actomyosin contraction pulls on an integrin that is either not attached, or attached to a very soft ECM.

Box 2: Molecular clutch theory. In essence, the molecular clutch theory considers how the activity of the actomyosin cytoskeleton and its link to the matrix through, actin, adaptor proteins, and integrins results in force transmission between the different elements, cytoskeletal dynamics, and cell migration. Because all the bonds in the actin-adaptor protein-integrin-ECM link can dynamically form and break, this is referred to as a "molecular clutch", in an analogy to the dynamic connection between shafts in a mechanical engine. Myosin contraction leads to a continuous flow of actin from cell edges towards their centre, known as retrograde flow. Actin polymerization further contributes to this flow by pushing against the plasma membrane ${ }^{214-217}$. Since the bonds linking actin to adaptor proteins, integrins, and the ECM continuously break and reform, actomyosin flows and forces are only partially transmitted, and slow down progressively from actin to integrins. Adaptor proteins exhibit lower speeds than actin, and in turn integrins exhibit even lower or sometimes even negligible speeds ${ }^{218}$. If one considers how force affects myosin contraction (by slowing it) ${ }^{16,219}$ and the binding dynamics between the different molecular players involved (by affecting unbinding rates as a catch or slip bond as described above), computational models can be built, which predict both the dynamics of cytosleketal movement and of force transmission ${ }^{220-226}$. If the respective speeds of actin retrograde flows and actin polymerization are compared, predictions for cell migration or cell spreading speeds can also be obtained ${ }^{220}{ }^{173,174}$. Such models can be further refined by also including the force sensitivity of mechanosensitive events in adaptor proteins (such as talin unfolding), leading to predictions of how external factors such as substrate rigidity or ECM ligand distribution regulate whether mechanosensing is triggered ${ }^{65,116,130}$.

Fig. 1 | Distinct levels of integrin regulation. Integrin transcription is regulated by multiple external signals such as ECM composition or signalling from growth factor receptors. Integrin $\alpha$ - and $\beta$-subunits heterodimerise in the ER/Golgi, mature through post-translational modifications, such as glycosylation, and are transported as an inactive heterodimer (maintained inactive by intraluminal calcium) to the plasma membrane $^{52}$. On the plasma membrane, extracellular magnesium enables integrin activation in conjunction with inside-out mechanisms ${ }^{14,17}$. Inside-out signals regulate displacement of integrin inactivators and enable talin binding to integrin $\beta$-tails, tightly controlling integrin affinity for ECM ligands ${ }^{25}$. In fibroblasts, FAK recruitment to integrins has been suggested to precede talin recruitment ${ }^{38}$. Subsequent ECM binding triggers integrin clustering, and their coupling via talin and vinculin to the actin cytoskeleton. Traffic of integrins regulates their cell surface availability. Integrins are constantly endocytosed from the plasma membrane and efficiently recycled, with a small subset of the receptors targeted to lysosomal degradation ${ }^{52}$. Integrins can be endocytosed via multiple different routes depending on the cell type, adhesion status, and cellular signalling pathways activated. Integrin cytoplasmic tails contain recognition motifs for clathrin adaptors, recruiting them as cargo for clathrin mediated endocytosis $(\mathrm{CME})^{49}$. Microtubules and CME have been implicated in 
focal adhesion turnover. Clathrin independent internalization mechanisms (CIE) include caveolin-dependent pathways and clathrin-independent carriers (CLICs) $)^{51,54,55}$.

Fig. 2 Force regulates integrin properties. a) Integrin ligand binding follows a catch-bond behaviour ${ }^{63}$. When force $(F)$ is below the optimal bond force $\left(F_{B}\right)$, the strength (lifetime) of the bond increases with force. When force exceeds $F_{B}$, bond lifetime decays with force. b) If a given force is applied to an adhesion site, further integrin clustering decreases the force applied to individual integrin dimers. This is energetically favourable since it decreases the applied strain, and could thus be promoted. c) Conformational changes in integrins (such as transitioning from the closed to the open state) can be induced by force, triggering outsidein integrin signalling ${ }^{64}$. d) The glycocalyx acts a natural barrier impairing integrin-ligand binding. Once integrins bind, they locally bend the membrane, decreasing integrin-ligand distance and promoting binding of further integrins (and thereby clustering) ${ }^{89}$. This membrane bending also exerts a tensile force on bound integrins due to its tendency to go back to its non-deformed state.

Fig. 3 | Integrins mediate response to ECM signals such as force, rigidity and ligand distribution. a) External forces or internal actomyosin contractility affect integrin response via catch bond mechanisms, integrin conformational changes, or clustering, as explained in fig. 2. Increased force can also extend integrin bond lifetime sufficiently, enabling talin unfolding (or other mechanosensitive events), allowing vinculin binding and the propagation of forces to the actin cytoskeleton ${ }^{65}$. These effects lead to the formation of focal adhesions and stress fibres, allowing forces to reach the nucleus through actomyosin contractility. Forces then influence nuclear shape, affecting nucleo-cytoplasmic transport and promoting the nuclear translocation of transcriptional regulators such as YAP/TAZ, ultimately altering gene expression ${ }^{137}$. b) Cells continuously pull on the ECM through integrins and actomyosin contractility. Higher rigidity results in increased force transmission between actin, integrins, and the ECM, resulting in the same effects described in a). c) Ligand distribution and density influence cellular mechanosensing through integrins by regulating integrin clustering and the forces exerted to single integrin molecules. Lower ligand density results in the concentration of forces in fewer integrin molecules ${ }^{130}$, leading to the same force-mediated effects described in a).

Fig.4 | Integrin-mediated regulation of cell migration and morphogenesis. a) Directional cell migration of individual cells and especially of cell clusters can be regulated by different gradients: Migration towards increased ECM rigidity (durotaxis), increased concentrations of ECM ligands (haptotaxis), or increased concentrations of soluble cues like growth factors of chemokines (chemotaxis) ${ }^{171,172}$. b) During puberty the ductal outgrowth of the mammary gland involves a process of collective migration, generating the ductal tree that fills the mammary fat pad. The ductal invasion is an integrin-dependent migration process led by a tip structure called the terminal end bud. The process is regulated by stromal rigidity and collagen alignment, features that are controlled by mammary gland stromal fibroblasts and integrin activation status ${ }^{175,178}$. c) During development, mechanical cues regulate the collective cell migration (CCM) of the Xenopus laevis neural crest cells (NC). As convergent extension (CE) progresses, the mesoderm stiffens prior to the onset of $\mathrm{NC}$ collective migration. This generates a mechanoresponsive signal through an integrin $\beta 1$-vinculin-talin axis,,triggering epithelial-to-mesenchymal transition (EMT) of NC and signalling for collective cell migration $(\mathrm{CCM})$ to proceed ${ }^{145}$. d) In carcinomas, fibroblasts in the tumor microenvironment become activated to give rise to cancer-associated fibroblasts (CAFs). These contribute mechanically to cancer cell migration and invasion via numerous distinct mechanisms. The more recently described ones include: increased fibronectin (FN) secretion and $\alpha v \beta 3$-integrin dependent alignment of the stroma, or increased contractility of activated CAFs and $\alpha 5 \beta 1$-induced alignment of fibronectin. CAF contractility is also crucial to remodel the BM, making it permissive for cancer cell invasion ${ }^{193-196}$. 


\section{Glossary}

TYPE I TRANSMEMBRANE PROTEINS

Proteins that span the cell membrane through a single transmembrane $\alpha$-helix.

RGD-MoTIF

Is a peptide sequence consisting of an Arginine, Glycine and an Aspartate. It is found in ECM molecules such as fibronectin and vitronectin and it serves as a binding site for integrins.

INSIDE-OUT INTEGRIN SIGNALING

Is the regulation of integrin activity by molecular interactions with the cytosolic domain of integrins.

INSIDE-OUT INTEGRIN SIGNALING

Is the regulation of integrin activity by molecular interactions with extracellular integrin ligands.

ADAPTOR PROTEIN.

In the context of integrin adhesions, "adaptor protein" refers to any of the several different types of recruited proteins that directly or indirectly link integrins to actin.

TALIN

A high molecular weight protein $(\sim 270 \mathrm{kDa})$ that links the integrin $\beta$ subunit to actin filaments, and promotes the assembly of focal adhesions (FAs). It consists of an N-terminal head region with the F0 and FERM domains, a flexible rod domain and a C-terminal dimerization sequence.

NASCENT ADHESIONS

Clusters of activated integrin molecules with sizes smaller than $1 \mu \mathrm{m}$. They either undergo fast disassembly or they progress to mature focal adhesions.

INSIDE-OUT INTEGRIN SIGNALING

Is the regulation of integrin activity by molecular interactions with the cytosolic domain of integrins.

OUTSIDE-IN INTEGRIN SIGNALING

Is the initiation of cell signaling cascades by integrins upon their interaction with ECM ligands.

FLUID-PHASE ENDOCYTOSIS

Is the continuous and non-specific uptake of extracellular fluid. This form of endocytosis is not mediated by a specific receptor or solute distribution.

BAR-PROTEIN

Proteins with a BAR (Bin/Amphiphysin/Rvs) domain. The special banana-shape conformation of BAR dimers creates a pocket of positive charges that could mediate phospholipid binding and curvature sensing or induction.

CELL TRACTION FORCE

Force per unit area that cells exert on a substrate.

GLYCOCALYX

Is a meshwork surrounding the cell membrane of many eukaryotic cells and bacteria. It consists of carbohydrates (mostly proteoglycans and glycoproteins) that extend out of the cell membrane.

FIBRILLAR ADHESIONS

Cell-ECM adhesion sites rich in $\alpha 5 \beta 1$ integrin and tensin. They are located towards the cell center and usually form along fibrils.

STRESS FIBRES 
Actin bundles rich in non-muscle myosin II and $\alpha$-actinin. They play an important role in force transmission and cellular contractility in non-muscle cells.

MATURE FOCAL ADHESIONS

Nascent adhesions mature to FAs upon tension generated by actomyosin contractility or external forces. This leads to protein recruitment and increase in FA length.

HAPTOTAXIS

Is the directional cell movement in response to a spatial gradient of ligand density.

DUROTAXIS

Is the directional cell movement in response to a stiffness gradient.

UROKINASE PLASMINOGEN ACTIVATOR RECEPTOR (UPAR)

Is a glycosyl-phosphatidylinositol (GPI)-anchored cell membrane receptor that acts as a receptor for urokinase plasminogen activator promoting plasmin formation.

TERMINAL END BUDS

Are bulb-shaped structures at the tip of a growing duct that direct its growth during breast morphogenesis.

CHEMOTAXIS

Is the directional cell movement in response to a spatial gradient of chemical signals.

CONVERGENT EXTENSION (CE)

Is the process of collective cell movement, by which tissues undergo elongation over one axis and narrowing over the other axis during embryonic development. 


\section{References}

1 Hynes, R. O. \& Yamada, K. M. Fibronectins: multifunctional modular glycoproteins. The Journal of Cell Biology 95, 369377, doi:10.1083/jcb.95.2.369 (1982).

2 Hynes, R. O. The emergence of integrins: a personal and historical perspective. Matrix biology : journal of the International Society for Matrix Biology 23, 333-340, doi:10.1016/j.matbio.2004.08.001 (2004).

3 Bissell, M. J., Hall, H. G. \& Parry, G. How does the extracellular matrix direct gene expression? J. Theor. Biol. 99, 31-68 (1982).

4 Winograd-Katz, S. E., Fassler, R., Geiger, B. \& Legate, K. R. The integrin adhesome: from genes and proteins to human disease. Nat. Rev. Mol. Cell Biol. 15, 273-288, doi:10.1038/nrm3769 (2014).

5 Hamidi, H. \& Ivaska, J. Every step of the way: integrins in cancer progression and metastasis. Nature Reviews Cancer 18, 533-548, doi:10.1038/s41568-018-0038-z (2018).

Humphries, J. D., Chastney, M. R., Askari, J. A. \& Humphries, M. J. Signal transduction via integrin adhesion complexes. Curr. Opin. Cell Biol. 56, 14-21, doi:10.1016/j.ceb.2018.08.004 (2018).

7 De Franceschi, N., Hamidi, H., Alanko, J., Sahgal, P. \& Ivaska, J. Integrin traffic - the update. J. Cell Sci. 128, 839-852, doi:10.1242/jcs.161653 (2015).

8 Sun, Z., Guo, S. S. \& Fassler, R. Integrin-mediated mechanotransduction. J. Cell Biol. 215, 445-456, doi:10.1083/jcb.201609037 (2016). Seetharaman, S. \& Etienne-Manneville, S. Integrin diversity brings specificity in mechanotransduction. Biol. Cell 110, 4964, doi:10.1111/boc.201700060 (2018).

1 doi:10.1091/mbc.E17-04-0228 (2017).

Bouvard, D., Pouwels, J., De Franceschi, N. \& Ivaska, J. Integrin inactivators: balancing cellular functions in vitro and in vivo. Nat. Rev. Mol. Cell Biol. 14, 430-442, doi:10.1038/nrm3599 (2013).

Hynes, R. O. Integrins: Bidirectional, allosteric signaling machines. Cell 110, 673-687 (2002).

Humphries, J. D., Byron, A. \& Humphries, M. J. Integrin ligands at a glance. J. Cell Sci. 119, 3901-3903, doi:10.1242/jcs.03098 (2006). integrins during their intracellular trafficking. J. Cell Sci. 124, 1672-1680, doi:10.1242/jcs.084483 (2011). Heino, J., Ignotz, R. A., Hemler, M. E., Crouse, C. \& Massagué, J. Regulation of cell adhesion receptors by transforming growth factor-beta. Concomitant regulation of integrins that share a common beta 1 subunit. J. Biol. Chem. 264, 380-388 (1989).

Xiong, J.-P. et al. Crystal Structure of the Extracellular Segment of Integrin $\alpha$ V $\beta 3$ in Complex with an Arg-Gly-Asp Ligand. Science 296, 151-155, doi:10.1126/science.1069040 (2002).

in outside-in and inside-out signaling. Cell 110, 599-611 (2002).

Takagi, J., Strokovich, K., Springer, T. A. \& Walz, T. Structure of integrin alpha5beta1 in complex with fibronectin. EMBO J. 22, 4607-4615, doi:10.1093/emboj/cdg445 (2003). Emsley, J., Knight, C. G., Farndale, R. W., Barnes, M. J. \& Liddington, R. C. Structural basis of collagen recognition by integrin alpha2beta1. Cell 101, 47-56, doi:10.1016/S0092-8674(00)80622-4 (2000). Lee, J. O., Rieu, P., Arnaout, M. A. \& Liddington, R. Crystal structure of the A domain from the alpha subunit of integrin CR3 (CD11b/CD18). Cell 80, 631-638 (1995). Cell. Dev. Biol. 27, 291-320, doi:10.1146/annurev-cellbio-092910-154017 (2011).

Gasparski, A. N. \& Beningo, K. A. Mechanoreception at the cell membrane: More than the integrins. Archives of biochemistry and biophysics 586, 20-26, doi:10.1016/j.abb.2015.07.017 (2015).

Ramovs, V., Te Molder, L. \& Sonnenberg, A. The opposing roles of laminin-binding integrins in cancer. Matrix biology : journal of the International Society for Matrix Biology 57-58, 213-243, doi:10.1016/j.matbio.2016.08.007 (2017). Tadokoro, S. et al. Talin binding to integrin beta tails: a final common step in integrin activation. Science 302, 103-106, doi:10.1126/science.1086652 (2003).

Shattil, S. J., Kim, C. \& Ginsberg, M. H. The final steps of integrin activation: the end game. Nature Reviews. Molecular Cell Biology 11, 288-300, doi:10.1038/nrm2871 (2010).

Rognoni, E., Ruppert, R. \& Fässler, R. The kindlin family: functions, signaling properties and implications for human disease. J. Cell. Sci. 129, 17-27, doi:10.1242/jcs.161190 (2016).

Böttcher, R. T. et al. Kindlin-2 recruits paxillin and Arp2/3 to promote membrane protrusions during initial cell spreading. $J$ Cell Biol 216, 3785-3798, doi:10.1083/jcb.201701176 (2017).

Georgiadou, M. \& Ivaska, J. Tensins: Bridging AMP-Activated Protein Kinase with Integrin Activation. Trends Cell Biol. 27, 703-711, doi:10.1016/j.tcb.2017.06.004 (2017). Georgiadou, M. et al. AMPK negatively regulates tensin-dependent integrin activity. J. Cell Biol. 216, 1107-1121, doi:10.1083/jcb.201609066 (2017).

McCleverty, C. J., Lin, D. C. \& Liddington, R. C. Structure of the PTB domain of tensin1 and a model for its recruitment to fibrillar adhesions. Protein Sci. 16, 1223-1229, doi:10.1110/ps.072798707 (2007). 
Bouin, A. P. et al. ICAP-1 monoubiquitylation coordinates matrix density and rigidity sensing for cell migration through ROCK2-MRCKalpha balance. J. Cell Sci. 130, 626-636, doi:10.1242/jcs.200139 (2017). Rossier, O. et al. Integrins $\beta 1$ and $\beta 3$ exhibit distinct dynamic nanoscale organizations inside focal adhesions. Nat. Cell Biol. 14, 1231, doi:10.1038/ncb2620 (2012).

Lee, H.-S., Lim, C. J., Puzon-McLaughlin, W., Shattil, S. J. \& Ginsberg, M. H. RIAM activates integrins by linking talin to ras GTPase membrane-targeting sequences. J. Biol. Chem. 284, 5119-5127, doi:10.1074/jbc.M807117200 (2009).

Zhu, L. et al. Structure of Raplb bound to talin reveals a pathway for triggering integrin activation. Nature Communications 8, 1744, doi:10.1038/s41467-017-01822-8 (2017).

De Franceschi, N. et al. ProLIF - quantitative integrin protein-protein interactions and synergistic membrane effects on proteoliposomes. J. Cell. Sci. 132, doi:10.1242/jcs.214270 (2018).

Lilja, J. et al. SHANK proteins limit integrin activation by directly interacting with Rap1 and R-Ras. Nat. Cell Biol. 19, 292-305, doi:10.1038/ncb3487 (2017).

Horton, E. R. et al. Modulation of FAK and Src adhesion signaling occurs independently of adhesion complex composition. J Cell Biol 212, 349-364, doi:10.1083/jcb.201508080 (2016). Biology 196, 223-232, doi:10.1083/jcb.201108078 (2012).

Horton, E. R. et al. Definition of a consensus integrin adhesome and its dynamics during adhesion complex assembly and disassembly. Nat. Cell Biol. 17, 1577-1587, doi:10.1038/ncb3257 (2015). Alanko, J. et al. Integrin endosomal signalling suppresses anoikis. Nat. Cell Biol. 17, 1412-1421, doi:10.1038/ncb3250 (2015).

Nader, G. P. F., Ezratty, E. J. \& Gundersen, G. G. FAK, talin and PIPKI $\gamma$ regulate endocytosed integrin activation to polarize focal adhesion assembly. Nat. Cell Biol. 18, 491-503, doi:10.1038/ncb3333 (2016). Shafaq-Zadah, M. et al. Persistent cell migration and adhesion rely on retrograde transport of $\beta(1)$ integrin. Nat. Cell Biol. 18, 54-64, doi:10.1038/ncb3287 (2016). Paul, N. R., Jacquemet, G. \& Caswell, P. T. Endocytic Trafficking of Integrins in Cell Migration. Curr. Biol. 25, R10921105, doi:10.1016/j.cub.2015.09.049 (2015).

Lobert, V. H. et al. Ubiquitination of alpha 5 beta 1 integrin controls fibroblast migration through lysosomal degradation of fibronectin-integrin complexes. Dev. Cell 19, 148-159, doi:10.1016/j.devcel.2010.06.010 (2010).

Steinberg, F., Heesom, K. J., Bass, M. D. \& Cullen, P. J. SNX17 protects integrins from degradation by sorting between lysosomal and recycling pathways. J Cell Biol 197, 219-230, doi:10.1083/jcb.201111121 (2012). McNally, K. E. et al. Retriever is a multiprotein complex for retromer-independent endosomal cargo recycling. Nat. Cell Biol. 19, 1214-1225, doi:10.1038/ncb3610 (2017). Ratcliffe, C. D. H., Sahgal, P., Parachoniak, C. A., Ivaska, J. \& Park, M. Regulation of Cell Migration and $\beta 1$ Integrin Trafficking by the Endosomal Adaptor GGA3. Traffic 17, 670-688, doi:10.1111/tra.12390 (2016). Böttcher, R. T. et al. Sorting nexin 17 prevents lysosom
Cell Biol. 14, 584-592, doi:10.1038/ncb2501 (2012). Ezratty, E. J., Bertaux, C., Marcantonio, E. E. \& Gundersen, G. G. Clathrin mediates integrin endocytosis for focal adhesion disassembly in migrating cells. The Journal of Cell Biology 187, 733-747, doi:10.1083/jcb.200904054 (2009). \\ Struct. Mol. Biol. 23, 172-179, doi:10.1038/nsmb.3161 (2016).}

51 Lakshminarayan, R. et al. Galectin-3 drives glycosphingolipid-dependent biogenesis of clathrin-independent carriers. Nat. Cell Biol. 16, 595-606, doi:10.1038/ncb2970 (2014). Moreno-Layseca, P., Icha, J., Hamidi, H. \& Ivaska, J. Integrin trafficking in cells and tissues. Nat. Cell Biol., In press, doi:https://doi.org/10.1038/s41556-018-0223-z (2019).

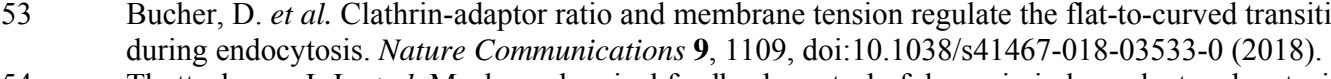

54 Thottacherry, J. J. et al. Mechanochemical feedback control of dynamin independent endocytosis modulates membrane tension in adherent cells. Nature Communications 9, 4217, doi:10.1038/s41467-018-06738-5 (2018). Holst, M. R. et al. Clathrin-Independent Endocyto
1905, doi:10.1016/j.celrep.2017.08.006 (2017). $\mathrm{Yu}$, C.-h. et al. Integrin-beta3 clusters recruit clathrin-mediated endocytic machinery in the absence of traction force. Nature Communications 6, 8672, doi:10.1038/ncomms9672 (2015). Schiller, H. B. et al. beta1- and alphav-class integrins cooperate to regulate myosin II during rigidity sensing of fibronectinbased microenvironments. Nat. Cell Biol. 15, 625-636, doi:10.1038/ncb2747 (2013). $\mathrm{Du}$, J. et al. Integrin activation and internalization on soft ECM as a mechanism of induction of stem cell differentiation by ECM elasticity. Proc Natl Acad Sci U S A 108, 9466-9471, doi:10.1073/pnas.1106467108 (2011).

doi:10.1038/s41570-017-0083 (2017).

60 Bell, G. I. Models for the specific adhesion of cells to cells. Science 200, 618-627 (1978).

61 Rakshit, S. \& Sivasankar, S. Biomechanics of cell adhesion: how force regulates the lifetime of adhesive bonds at the single molecule level. Physical Chemistry Chemical Physics 16, 2211-2223, doi:10.1039/C3CP53963F (2014). 048101, doi:10.1103/PhysRevLett.104.048101 (2010). 

its ligand. J. Cell Biol. 185, 1275-1284 (2009).

64 Chen, Y., Lee, H., Tong, H., Schwartz, M. \& Zhu, C. Force regulated conformational change of integrin $\alpha$ V $\beta 3$. Matrix Biology, doi:http://dx.doi.org/10.1016/j.matbio.2016.07.002 (2016).

65 Elosegui-Artola, A. et al. Mechanical regulation of a molecular clutch defines force transmission and transduction in response to matrix rigidity. Nat. Cell Biol. 18, 540-548, doi:10.1038/ncb3336 (2016).

66 Chen, W., Lou, J. \& Zhu, C. Forcing switch from short- to intermediate- and long-lived states of the alphaA domain generates LFA-1/ICAM-1 catch bonds. J. Biol. Chem. 285, 35967-35978, doi:10.1074/jbc.M110.155770 (2010). Choi, Y. I. et al. Dynamic control of beta1 integrin adhesion by the plexinD1-sema3E axis. PNAS 111, 379-384, doi:10.1073/pnas.1314209111 (2014).

Rosetti, F. et al. A Lupus-Associated Mac-1 Variant Has Defects in Integrin Allostery and Interaction with Ligands under Force. Cell reports, doi:10.1016/j.celrep.2015.02.037 (2015). Friedland, J. C., Lee, M. H. \& Boettiger, D. Mechanically Activated Integrin Switch Controls alpha(5)beta(1) Function. Science 323, 642-644 (2009).

70 Benito-Jardon, M. et al. The fibronectin synergy site re-enforces cell adhesion and mediates a crosstalk between integrin classes. eLife 6, doi:10.7554/eLife.22264 (2017).

71 Zhu, J., Zhu, J. \& Springer, T. A. Complete integrin headpiece opening in eight steps. J. Cell Biol. 201, 1053-1068, doi:10.1083/jcb.201212037 (2013). Chakrabarti, S., Hinczewski, M. \& Thirumalai, D. Plasticity of hydrogen bond networks regulates mechanochemistry of cell adhesion complexes. PNAS 111, 9048-9053, doi:10.1073/pnas.1405384111 (2014). Chakrabarti, S., Hinczewski, M. \& Thirumalai, D. Phenomenological and microscopic theories for catch bonds. Journal of structural biology 197, 50-56, doi:10.1016/j.jsb.2016.03.022 (2017).

Rakshit, S., Zhang, Y., Manibog, K., Shafraz, O. \& Sivasankar, S. Ideal, catch, and slip bonds in cadherin adhesion. PNAS 109, 18815-18820, doi:10.1073/pnas.1208349109 (2012). Fennewald, S. M., Kantara, C., Sastry, S. K. \& Resto, V. A. Laminin interactions with head and neck cancer cells under low fluid shear conditions lead to integrin activation and binding. J. Biol. Chem. 287, 21058-21066, doi:10.1074/jbc.M112.360313 (2012). Puklin-Faucher, E. \& Vogel, V. Integrin activation dynamics between the RGD-binding site and the headpiece hinge. $J$. Biol. Chem. 284, 36557-36568, doi:10.1074/jbc.M109.041194 (2009).

77 Zhu, J. et al. Structure of a complete integrin ectodomain in a physiologic resting state and activation and deactivation by applied forces. Mol. Cell 32, 849-861, doi:10.1016/j.molcel.2008.11.018 (2008). Chen, W. et al. Molecular dynamics simulations of forced unbending of integrin alpha(v)beta(3). PLoS Comput Biol 7, e1001086, doi:10.1371/journal.pcbi.1001086 (2011). Chen, W., Lou, J., Evans, E. A. \& Zhu, C. Observing force-regulated conformational changes and ligand dissociation from a single integrin on cells. J. Cell Biol. 199, 497-512, doi:10.1083/jcb.201201091 (2012).

80 Comrie, W. A., Babich, A. \& Burkhardt, J. K. F-actin flow drives affinity maturation and spatial organization of LFA-1 at the immunological synapse. J. Cell Biol. 208, 475-491, doi:10.1083/jcb.201406121 (2015).

81 Nordenfelt, P., Elliott, H. L. \& Springer, T. A. Coordinated integrin activation by actin-dependent force during T-cell migration. Nat. Commun. 7, 13119, doi:10.1038/ncomms 13119

https:/www.nature.com/articles/ncomms13119\#supplementary-information (2016).

82 Kong, F. et al. Cyclic mechanical reinforcement of integrin-ligand interactions. Mol. Cell 49, 1060-1068, doi:10.1016/j.molcel.2013.01.015 (2013).

83 Gonzalez-Tarrago, V. et al. Binding of ZO-1 to alpha5beta1 integrins regulates the mechanical properties of alpha5beta1fibronectin links. Mol. Biol. Cell. 28, 1847-1852, doi:10.1091/mbc.E17-01-0006 (2017).

84 Li, J. \& Springer, T. A. Integrin extension enables ultrasensitive regulation by cytoskeletal force. PNAS 114, 4685-4690, doi:10.1073/pnas.1704171114 (2017). Shemesh, T., Geiger, B., Bershadsky, A. D. \& Kozlov, M. M. Focal adhesions as mechanosensors: A physical mechanism. PNAS 102, 12383-12388, doi:10.1073/pnas.0500254102 (2005). Cao, X. et al. A Chemomechanical Model of Matrix and Nuclear Rigidity Regulation of Focal Adhesion Size. Biophys. J. 109, 1807-1817, doi:10.1016/j.bpj.2015.08.048 (2015). Chaudhuri, O. et al. Extracellular matrix stiffness and composition jointly regulate the induction of malignant phenotypes in mammary epithelium. Nat Mater 13, 970-978, doi:10.1038/nmat4009 (2014). Paszek, M. J., Boettiger, D., Weaver, V. M. \& Hammer, D. A. Integrin clustering is driven by mechanical resistance from the glycocalyx and the substrate. PLoS Comput Biol 5, e1000604, doi:10.1371/journal.pcbi.1000604 (2009). Paszek, M. J. et al. The cancer glycocalyx mechanically primes integrin-mediated growth and survival. Nature 511, 319325, doi:10.1038/nature13535 (2014). Eng, E. T., Smagghe, B. J., Walz, T. \& Springer, T. A. Intact $\alpha$ Ilb $\beta 3$ Integrin Is Extended after Activation as Measured by Solution X-ray Scattering and Electron Microscopy. J. Biol. Chem. 286, 35218-35226, doi:10.1074/jbc.M111.275107 (2011).

91 Elosegui-Artola, A., Trepat, X. \& Roca-Cusachs, P. Control of mechanotransduction by molecular clutch dynamics. Trends Cell Biol., In press (2018).

92 Roca-Cusachs, P., Conte, V. \& Trepat, X. Quantifying forces in cell biology. Nat. Cell Biol. 19, 742-751, doi:10.1038/ncb3564 (2017). 
Humphrey, J. D., Schwartz, M. A., Tellides, G. \& Milewicz, D. M. Role of mechanotransduction in vascular biology: focus on thoracic aortic aneurysms and dissections. Circ Res 116, 1448-1461, doi:10.1161/CIRCRESAHA.114.304936 (2015).

94 Waters, C. M., Roan, E. \& Navajas, D. Mechanobiology in lung epithelial cells: measurements, perturbations, and responses. Comprehensive Physiology 2, 1-29, doi:10.1002/cphy.c100090 (2012). Paszek, M. J. \& Weaver, V. M. The tension mounts: mechanics meets morphogenesis and malignancy. J Mammary Gland Biol Neoplasia 9, 325-342, doi:10.1007/s10911-004-1404-x (2004). and in patients with an unstable detrusor or bladder outlet obstruction. BJU International 92, 584-588, doi:doi:10.1046/j.1464-410X.2003.04420.x (2003). Mol. Cell Biol. 12, 308-319, doi:10.1038/nrm3112 (2011). Single Integrin Molecules in Living Cells. Nano Lett. 13, 3985-3989, doi:10.1021/nl4005145 (2013).

Zhang, Y., Ge, C., Zhu, C. \& Salaita, K. DNA-based digital tension probes reveal integrin forces during early cell adhesion. Nat. Commun. 5, 5167, doi:10.1038/ncomms6167 (2014). Blakely, B. L. et al. A DNA-based molecular probe for optically reporting cellular traction forces. Nat. Methods 11, 12291232, doi:10.1038/nmeth.3145 (2014).

101 Chang, A. C. et al. Single Molecule Force Measurements in Living Cells Reveal a Minimally Tensioned Integrin State. ACS nano 10, 10745-10752, doi:10.1021/acsnano.6b03314 (2016).

102 Wang, X. \& Ha, T. Defining single molecular forces required to activate integrin and notch signaling. Science 340, 991994, doi:10.1126/science.1231041 (2013). Rahil, Z. et al. Nanoscale Mechanics Guides Cellular Decision Making. Integrative Biology, doi:10.1039/C6IB00113K (2016).

104 Galior, K., Liu, Y., Yehl, K., Vivek, S. \& Salaita, K. Titin-Based Nanoparticle Tension Sensors Map High-Magnitude Integrin Forces within Focal Adhesions. Nano Lett. 16, 341-348, doi:10.1021/acs.nanolett.5b03888 (2016).

105 Zhang, Y. et al. Platelet integrins exhibit anisotropic mechanosensing and harness piconewton forces to mediate platelet aggregation. PNAS 115, 325-330, doi:10.1073/pnas.1710828115 (2018). whereas $\alpha v \beta 3$ and talin enable mechanotransduction. PNAS 106, 16245-16250 (2009).

Roca-Cusachs, P. et al. Integrin-dependent force transmission to the extracellular matrix by alpha-actinin triggers adhesion maturation. PNAS 110, E1361-E1370, doi:1220723110 [pii];10.1073/pnas.1220723110 [doi] (2013).

Jiang, G. Y., Giannone, G., Critchley, D. R., Fukumoto, E. \& Sheetz, M. P. Two-piconewton slip bond between fibronectin and the cytoskeleton depends on talin. Nature 424, 334-337 (2003).

Strohmeyer, N., Bharadwaj, M., Costell, M., Fassler, R. \& Muller, D. J. Fibronectin-bound alpha5beta1 integrins sense load and signal to reinforce adhesion in less than a second. Nat Mater, doi:10.1038/nmat5023 (2017).

Riveline, D. et al. Focal contacts as mechanosensors: Externally applied local mechanical force induces growth of focal contacts by an mDia1-dependent and ROCK-independent mechanism. J. Cell Biol. 153, 1175-1185 (2001).

Galbraith, C. G., Yamada, K. M. \& Sheetz, M. P. The relationship between force and focal complex development. J. Cell Biol. 159, 695-705 (2002).

Paszek, M. J. et al. Tensional homeostasis and the malignant phenotype. Cancer Cell 8, 241-254, doi:10.1016/j.ccr.2005.08.010 (2005).

Hirata, H., Sokabe, M. \& Lim, C. T. Molecular mechanisms underlying the force-dependent regulation of actin-to-ECM linkage at the focal adhesions. Progress in molecular biology and translational science 126, 135-154, doi:10.1016/B978-012-394624-9.00006-3 (2014).

Schiller, H. B. \& Fassler, R. Mechanosensitivity and compositional dynamics of cell-matrix adhesions. EMBO reports $\mathbf{1 4}$, 509-519, doi:10.1038/embor.2013.49 (2013).

Changede, R., Xu, X., Margadant, F. \& Sheetz, M. P. Nascent Integrin Adhesions Form on All Matrix Rigidities after Integrin Activation. Dev. Cell 35, 614-621, doi:10.1016/j.devcel.2015.11.001 (2015).

Elosegui-Artola, A. et al. Rigidity sensing and adaptation through regulation of integrin types. Nat Mater 13, 631-637, doi:10.1038/nmat3960 (2014).

Brockman, J. M. et al. Mapping the 3D orientation of piconewton integrin traction forces. Nat. Methods 15, 115-118, doi:10.1038/nmeth.4536 (2018).

Swaminathan, V. et al. Actin retrograde flow actively aligns and orients ligand-engaged integrins in focal adhesions. PNAS 114, 10648-10653, doi:10.1073/pnas.1701136114 (2017).

Nordenfelt, P. et al. Direction of actin flow dictates integrin LFA-1 orientation during leukocyte migration. Nat. Commun. 8, 2047, doi:10.1038/s41467-017-01848-y (2017).

del Rio, A. et al. Stretching Single Talin Rod Molecules Activates Vinculin Binding. Science 323, 638-641 (2009).

Yao, M. et al. Mechanical activation of vinculin binding to talin locks talin in an unfolded conformation. Sci. Rep. 4, 4610, doi:10.1038/srep04610 (2014).

Zhang, X. et al. Talin depletion reveals independence of initial cell spreading from integrin activation and traction. Nat. Cell Biol. 10, 1062-1068 (2008).

Bharadwaj, M. et al. alphaV-class integrins exert dual roles on alpha5beta1 integrins to strengthen adhesion to fibronectin. Nat. Commun. 8, 14348, doi:10.1038/ncomms14348 (2017). 
124 Balcioglu, H. E., van Hoorn, H., Donato, D. M., Schmidt, T. \& Danen, E. H. The integrin expression profile modulates orientation and dynamics of force transmission at cell-matrix adhesions. J. Cell Sci. 128, 1316-1326, doi:10.1242/jcs.156950 (2015).

125 Seong, J. et al. Distinct biophysical mechanisms of focal adhesion kinase mechanoactivation by different extracellular matrix proteins. PNAS 110, 19372-19377, doi:10.1073/pnas.1307405110 (2013).

126 Stricker, J., Aratyn-Schaus, Y., Oakes, P. W. \& Gardel, M. L. Spatiotemporal constraints on the force-dependent growth of focal adhesions. Biophys. J. 100, 2883-2893, doi:10.1016/j.bpj.2011.05.023 (2011).

127 Choi, C. K. et al. Actin and alpha-actinin orchestrate the assembly and maturation of nascent adhesions in a myosin II motor-independent manner. Nat. Cell Biol. 10, 1039-1050 (2008).

Oakes, P. W., Beckham, Y., Stricker, J. \& Gardel, M. L. Tension is required but not sufficient for focal adhesion maturation without a stress fiber template. J. Cell Biol. 196, 363-374, doi:10.1083/jcb.201107042 (2012).

129 Oakes, P. W. et al. Lamellipodium is a myosin-independent mechanosensor. PNAS 115, 2646-2651, doi:10.1073/pnas.1715869115 (2018).

130 Oria, R. et al. Force loading explains spatial sensing of ligands by cells. Nature 552, 219-224, doi:10.1038/nature24662 (2017).

131 Pasapera, A. M., Schneider, I. C., Rericha, E., Schlaepfer, D. D. \& Waterman, C. M. Myosin II activity regulates vinculin recruitment to focal adhesions through FAK-mediated paxillin phosphorylation. J. Cell Biol. 188, 877-890, doi:10.1083/jcb.200906012 (2010). Plotnikov, S. V., Pasapera, A. M., Sabass, B. \& Waterman, C. M. Force fluctuations within focal adhesions mediate ECMrigidity sensing to guide directed cell migration. Cell 151, 1513-1527, doi:10.1016/j.cell.2012.11.034 (2012).

133 Guilluy, C. et al. The Rho GEFs LARG and GEF-H1 regulate the mechanical response to force on integrins. Nat. Cell Biol. 13, 722-727, doi:10.1038/ncb2254 (2011).

134 Pawlowski, R., Rajakyla, E. K., Vartiainen, M. K. \& Treisman, R. An actin-regulated importin alpha/beta-dependent extended bipartite NLS directs nuclear import of MRTF-A. EMBO J. 29, 3448-3458, doi:10.1038/emboj.2010.216 (2010). Chang, L. et al. The SWI/SNF complex is a mechanoregulated inhibitor of YAP and TAZ. Nature 563, 265-269, doi:10.1038/s41586-018-0658-1 (2018).

136 Kirby, T. J. \& Lammerding, J. Emerging views of the nucleus as a cellular mechanosensor. Nat. Cell Biol. 20, 373-381, doi:10.1038/s41556-018-0038-y (2018).

137 Elosegui-Artola, A. et al. Force Triggers YAP Nuclear Entry by Regulating Transport across Nuclear Pores. Cell 171, 13971410 e1314, doi:10.1016/j.cell.2017.10.008 (2017).

138 Tajik, A. et al. Transcription upregulation via force-induced direct stretching of chromatin. Nat Mater 15, 1287-1296, doi:10.1038/nmat4729 (2016).

139 Swift, J. et al. Nuclear lamin-A scales with tissue stiffness and enhances matrix-directed differentiation. Science 341 , 1240104, doi:10.1126/science.1240104 (2013).

140 Ihalainen, T. O. et al. Differential basal-to-apical accessibility of lamin A/C epitopes in the nuclear lamina regulated by changes in cytoskeletal tension. Nat Mater 14, 1252-1261, doi:10.1038/nmat4389 (2015).

141 Guilluy, C. et al. Isolated nuclei adapt to force and reveal a mechanotransduction pathway in the nucleus. Nat. Cell Biol. 16, 376-381, doi:10.1038/ncb2927 (2014).

142 Bonnans, C., Chou, J. \& Werb, Z. Remodelling the extracellular matrix in development and disease. Nature Reviews Molecular Cell Biology 15, 786, doi:10.1038/nrm3904 (2014).

143 Butcher, D. T., Alliston, T. \& Weaver, V. M. A tense situation: forcing tumour progression. Nat. Rev. Cancer 9, 108-122, doi:10.1038/nrc2544 (2009).

144 Handorf, A. M., Zhou, Y., Halanski, M. A. \& Li, W. J. Tissue stiffness dictates development, homeostasis, and disease progression. Organogenesis 11, 1-15, doi:10.1080/15476278.2015.1019687 (2015).

145 Barriga, E. H., Franze, K., Charras, G. \& Mayor, R. Tissue stiffening coordinates morphogenesis by triggering collective cell migration in vivo. Nature 554, 523-527, doi:10.1038/nature25742 (2018).

146 Northey, J. J., Przybyla, L. \& Weaver, V. M. Tissue Force Programs Cell Fate and Tumor Aggression. Cancer discovery 7, 1224-1237, doi:10.1158/2159-8290.CD-16-0733 (2017).

147 Faurobert, E., Bouin, A. P. \& Albiges-Rizo, C. Microenvironment, tumor cell plasticity, and cancer. Current opinion in oncology 27, 64-70, doi:10.1097/CCO.0000000000000154 (2015).

148 Wu, Z., Plotnikov, S. V., Moalim, A. Y., Waterman, C. M. \& Liu, J. Two Distinct Actin Networks Mediate Traction Oscillations to Confer Focal Adhesion Mechanosensing. Biophys. J. 112, 780-794, doi:10.1016/j.bpj.2016.12.035 (2017).

149 Wolfenson, H. et al. Tropomyosin controls sarcomere-like contractions for rigidity sensing and suppressing growth on soft matrices. Nat. Cell Biol. 18, 33-42, doi:10.1038/ncb3277 (2016).

150 Ghassemi, S. et al. Cells test substrate rigidity by local contractions on sub-micrometer pillars. PNAS 109, 5328-5333 (2012).

151 Meacci, G. et al. alpha-actinin links ECM rigidity sensing contractile units with periodic cell edge retractions. Mol. Biol. Cell., doi:10.1091/mbc.E16-02-0107 (2016).

152 Chaudhuri, O. et al. Substrate stress relaxation regulates cell spreading. Nat. Commun. 6, 6364, doi:10.1038/ncomms7365 (2015).

153 Wisdom, K. M. et al. Matrix mechanical plasticity regulates cancer cell migration through confining microenvironments. Nat. Commun. 9, 4144, doi:10.1038/s41467-018-06641-z (2018).

154 Gong, Z. et al. Matching material and cellular timescales maximizes cell spreading on viscoelastic substrates. PNAS 115, E2686-E2695, doi:10.1073/pnas.1716620115 (2018). 
Bennett, M. et al. Molecular clutch drives cell response to surface viscosity. PNAS, doi:10.1073/pnas.1710653115 (2018). Gross, J. \& Nagai, Y. Specific degradation of the collagen molecule by tadpole collagenolytic enzyme. PNAS 54, $1197-$ 1204 (1965).

Stark, M. \& Kühn, K. The Properties of Molecular Fragments Obtained on Treating Calfskin Collagen with Collagenase from Clostridium histolyticum. Eur. J. Biochem. 6, 534-541, doi:doi:10.1111/j.1432-1033.1968.tb00477.x (1968).

Taubenberger, A. V., Woodruff, M. A., Bai, H., Muller, D. J. \& Hutmacher, D. W. The effect of unlocking RGD-motifs in collagen I on pre-osteoblast adhesion and differentiation. Biomaterials 31, 2827-2835, doi:10.1016/j.biomaterials.2009.12.051 (2010).

Ortiz Franyuti, D., Mitsi, M. \& Vogel, V. Mechanical Stretching of Fibronectin Fibers Upregulates Binding of Interleukin7. Nano Lett. 18, 15-25, doi:10.1021/acs.nanolett.7b01617 (2018).

Deng, Z. J., Liang, M., Monteiro, M., Toth, I. \& Minchin, R. F. Nanoparticle-induced unfolding of fibrinogen promotes Mac-1 receptor activation and inflammation. Nature nanotechnology 6, 39-44, doi:10.1038/nnano.2010.250 (2011). Arnold, M. et al. Activation of integrin function by nanopatterned adhesive interfaces. Chemphyschem 5, 383-388 (2004). Cavalcanti-Adam, E. A. et al. Lateral spacing of integrin ligands influences cell spreading and focal adhesion assembly. Eur. J. Cell Biol. 85, 219-224, doi:10.1016/j.ejcb.2005.09.011 (2006).

Altrock, E., Muth, C. A., Klein, G., Spatz, J. P. \& Lee-Thedieck, C. The significance of integrin ligand nanopatterning on lipid raft clustering in hematopoietic stem cells. Biomaterials 33, 3107-3118, doi:10.1016/j.biomaterials.2012.01.002 (2012).

Amschler, K., Erpenbeck, L., Kruss, S. \& Schon, M. P. Nanoscale integrin ligand patterns determine melanoma cell behavior. ACS nano 8,9113-9125, doi:10.1021/nn502690b (2014).

Cavalcanti-Adam, E. A. et al. Cell spreading and focal adhesion dynamics are regulated by spacing of integrin ligands. Biophys. J. 92, 2964-2974 (2007).

Huang, J. et al. Impact of order and disorder in RGD nanopatterns on cell adhesion. Nano Lett. 9, 1111-1116, doi:10.1021/nl803548b (2009).

Schvartzman, M. et al. Nanolithographic Control of the Spatial Organization of Cellular Adhesion Receptors at the SingleMolecule Level. Nano Lett. 11, 1306-1312 (2011).

Liu, Y. et al. Nanoparticle tension probes patterned at the nanoscale: impact of integrin clustering on force transmission. Nano Lett. 14, 5539-5546, doi:10.1021/n1501912g (2014).

Wipff, P. J., Rifkin, D. B., Meister, J. J. \& Hinz, B. Myofibroblast contraction activates latent TGF-beta1 from the extracellular matrix. J. Cell Biol. 179, 1311-1323, doi:10.1083/jcb.200704042 (2007).

Sarrazy, V. et al. Integrins alphavbeta5 and alphavbeta3 promote latent TGF-beta1 activation by human cardiac fibroblast contraction. Cardiovascular research 102, 407-417, doi:10.1093/cvr/cvu053 (2014).

Haeger, A., Wolf, K., Zegers, M. M. \& Friedl, P. Collective cell migration: guidance principles and hierarchies. Trends in Cell Biology 25, 556-566, doi:10.1016/j.tcb.2015.06.003 (2015).

Lo, C. M., Wang, H. B., Dembo, M. \& Wang, Y. L. Cell movement is guided by the rigidity of the substrate. Biophys. J. 79, 144-152 (2000).

Bangasser, B. L. et al. Shifting the optimal stiffness for cell migration. Nat. Commun. 8, 15313, doi:10.1038/ncomms15313 (2017).

Sunyer, R. et al. Collective cell durotaxis emerges from long-range intercellular force transmission. Science 353, $1157-$ 1161, doi:10.1126/science.aaf7119 (2016).

Schedin, P. \& Keely, P. J. Mammary Gland ECM Remodeling, Stiffness, and Mechanosignaling in Normal Development and Tumor Progression. Cold Spring Harb Perspect Biol 3, doi:10.1101/cshperspect.a003228 (2011).

Monaghan, P., Warburton, M. J., Perusinghe, N. \& Rudland, P. S. Topographical arrangement of basement membrane proteins in lactating rat mammary gland: comparison of the distribution of type IV collagen, laminin, fibronectin, and Thy-1 at the ultrastructural level. Proc Natl Acad Sci U S A 80, 3344-3348 (1983).

Ingman, W. V., Wyckoff, J., Gouon-Evans, V., Condeelis, J. \& Pollard, J. W. Macrophages promote collagen fibrillogenesis around terminal end buds of the developing mammary gland. Dev. Dyn. 235, 3222-3229, doi:10.1002/dvdy.20972 (2006).

Peuhu, E. et al. SHARPIN regulates collagen architecture and ductal outgrowth in the developing mouse mammary gland. EMBO J. 36, 165-182, doi:10.15252/embj.201694387 (2017).

Brownfield, D. G. et al. Patterned collagen fibers orient branching mammary epithelium through distinct signaling modules. Curr. Biol. 23, 703-709, doi:10.1016/j.cub.2013.03.032 (2013).

Lopez, J. I., Kang, I., You, W.-K., McDonald, D. M. \& Weaver, V. M. In situ force mapping of mammary gland transformation. Integr Biol (Camb) 3, 910-921, doi:10.1039/c1ib00043h (2011).

Conklin, M. W. et al. Aligned collagen is a prognostic signature for survival in human breast carcinoma. Am. J. Pathol. 178, 1221-1232, doi:10.1016/j.ajpath.2010.11.076 (2011).

Sauka-Spengler, T. \& Bronner-Fraser, M. A gene regulatory network orchestrates neural crest formation. Nat. Rev. Mol. Cell Biol. 9, 557-568, doi:10.1038/nrm2428 (2008).

Kerosuo, L. \& Bronner-Fraser, M. What's bad in cancer is good in the embryo: Importance of EMT in neural crest development. Semin Cell Dev Biol 23, 320-332, doi:10.1016/j.semcdb.2012.03.010 (2012).

Naba, A. et al. The matrisome: in silico definition and in vivo characterization by proteomics of normal and tumor extracellular matrices. Mol. Cell Proteomics 11, M111.014647, doi:10.1074/mcp.M111.014647 (2012).

Friedl, P. \& Alexander, S. Cancer invasion and the microenvironment: plasticity and reciprocity. Cell 147, 992-1009, doi:10.1016/j.cell.2011.11.016 (2011). 
186 McDonald, J. A., Kelley, D. G. \& Broekelmann, T. J. Role of fibronectin in collagen deposition: Fab' to the gelatin-binding domain of fibronectin inhibits both fibronectin and collagen organization in fibroblast extracellular matrix. J Cell Biol 92, 485-492 (1982).

187 Lemmon, C. A., Chen, C. S. \& Romer, L. H. Cell traction forces direct fibronectin matrix assembly. Biophys. J. 96, 729738, doi:10.1016/j.bpj.2008.10.009 (2009).

188 Oudin, M. J. \& Weaver, V. M. Physical and Chemical Gradients in the Tumor Microenvironment Regulate Tumor Cell Invasion, Migration, and Metastasis. Cold Spring Harb. Symp. Quant. Biol. 81, 189-205, doi:10.1101/sqb.2016.81.030817 (2016).

189 Barbazán, J. \& Matic Vignjevic, D. Cancer associated fibroblasts: is the force the path to the dark side? Curr. Opin. Cell Biol. 56, 71-79, doi:10.1016/j.ceb.2018.09.002 (2018).

190 Totaro, A., Panciera, T. \& Piccolo, S. YAP/TAZ upstream signals and downstream responses. Nat. Cell Biol. 20, 888-899, doi:10.1038/s41556-018-0142-z (2018).

191 De Wever, O. et al. Tenascin-C and SF/HGF produced by myofibroblasts in vitro provide convergent pro-invasive signals to human colon cancer cells through RhoA and Rac. FASEB J. 18, 1016-1018, doi:10.1096/fj.03-1110fje (2004).

192 Oudin, M. J. et al. Tumor Cell-Driven Extracellular Matrix Remodeling Drives Haptotaxis during Metastatic Progression. Cancer Discov 6, 516-531, doi:10.1158/2159-8290.CD-15-1183 (2016).

193 Erdogan, B. et al. Cancer-associated fibroblasts promote directional cancer cell migration by aligning fibronectin. J. Cell Biol. 216, 3799-3816, doi:10.1083/jcb.201704053 (2017).

194 Attieh, Y. et al. Cancer-associated fibroblasts lead tumor invasion through integrin-beta3-dependent fibronectin assembly. J. Cell Biol. 216, 3509-3520, doi:10.1083/jcb.201702033 (2017).

195 Castro-Castro, A. et al. Cellular and Molecular Mechanisms of MT1-MMP-Dependent Cancer Cell Invasion. Annu. Rev. Cell Dev. Biol. 32, 555-576, doi:10.1146/annurev-cellbio-111315-125227 (2016).

196 Glentis, A. et al. Cancer-associated fibroblasts induce metalloprotease-independent cancer cell invasion of the basement membrane. Nature Communications 8, 924, doi:10.1038/s41467-017-00985-8 (2017).

197 Lee, J. L. \& Streuli, C. H. Integrins and epithelial cell polarity. J. Cell. Sci. 127, 3217-3225, doi:10.1242/jcs.146142 (2014).

198 Yang, J. et al. Twist, a master regulator of morphogenesis, plays an essential role in tumor metastasis. Cell 117, 927-939, doi:10.1016/j.cell.2004.06.006 (2004).

$199 \mathrm{Xu}, \mathrm{Y}$. et al. Twist1 promotes breast cancer invasion and metastasis by silencing Foxa1 expression. Oncogene 36, 11571166, doi:10.1038/onc.2016.286 (2017).

200 Yang, J. et al. Twist induces epithelial-mesenchymal transition and cell motility in breast cancer via ITGB1-FAK/ILK signaling axis and its associated downstream network. Int. J. Biochem. Cell Biol. 71, 62-71, doi:10.1016/j.biocel.2015.12.004 (2016).

201 Sirka, O. K., Shamir, E. R. \& Ewald, A. J. Myoepithelial cells are a dynamic barrier to epithelial dissemination. J Cell Biol 217, 3368-3381, doi:10.1083/jcb.201802144 (2018).

202 Wei, S. C. et al. Matrix stiffness drives epithelial-mesenchymal transition and tumour metastasis through a TWIST1-G3BP2 mechanotransduction pathway. Nat. Cell Biol. 17, 678-688, doi:10.1038/ncb3157 (2015).

203 Chaurasia, P. et al. A region in urokinase plasminogen receptor domain III controlling a functional association with alpha5beta1 integrin and tumor growth. J. Biol. Chem. 281, 14852-14863, doi:10.1074/jbc.M512311200 (2006).

204 Shibue, T., Brooks, M. W. \& Weinberg, R. A. An integrin-linked machinery of cytoskeletal regulation that enables experimental tumor initiation and metastatic colonization. Cancer Cell 24, 481-498, doi:10.1016/j.ccr.2013.08.012 (2013).

205 Barkan, D. et al. Metastatic growth from dormant cells induced by a col-I-enriched fibrotic environment. Cancer Res. 70, 5706-5716, doi:10.1158/0008-5472.CAN-09-2356 (2010).

206 Barkan, D. et al. Inhibition of metastatic outgrowth from single dormant tumor cells by targeting the cytoskeleton. Cancer Res. 68, 6241-6250, doi:10.1158/0008-5472.CAN-07-6849 (2008).

207 Weaver, V. M. et al. Reversion of the malignant phenotype of human breast cells in three-dimensional culture and in vivo by integrin blocking antibodies. J Cell Biol 137, 231-245 (1997).

208 Albrengues, J. et al. Neutrophil extracellular traps produced during inflammation awaken dormant cancer cells in mice. Science 361, doi:10.1126/science.aao4227 (2018).

209 Reynolds, A. R. et al. Stimulation of tumor growth and angiogenesis by low concentrations of RGD-mimetic integrin inhibitors. Nat. Med. 15, 392, doi:10.1038/nm.1941

https://www.nature.com/articles/nm.1941\#supplementary-information (2009)

210 Cavallaro, U. \& Christofori, G. Cell adhesion in tumor invasion and metastasis: loss of the glue is not enough. Biochim. Biophys. Acta 1552, 39-45 (2001).

211 Austen, K. et al. Extracellular rigidity sensing by talin isoform-specific mechanical linkages. Nat. Cell Biol. 17, 1597-1606, doi:10.1038/ncb3268 (2015).

212 Kumar, A. et al. Talin tension sensor reveals novel features of focal adhesion force transmission and mechanosensitivity. $J$. Cell Biol. 213, 371-383, doi:10.1083/jcb.201510012 (2016).

213 Grashoff, C. et al. Measuring mechanical tension across vinculin reveals regulation of focal adhesion dynamics. Nature 466, 263-266 (2010).

214 Lin, C.-H. \& Forscher, P. Growth cone advance is inversely proportional to retrograde F-actin flow. Neuron 14, 763-771, doi:10.1016/0896-6273(95)90220-1.

215 Forscher, P. \& Smith, S. J. Actions of cytochalasins on the organization of actin filaments and microtubules in a neuronal growth cone. J.Cell Biol. 107, 1505-1516 (1988). 
216 Wang, Y. L. Exchange of actin subunits at the leading edge of living fibroblasts: possible role of treadmilling. The Journal of Cell Biology 101, 597-602, doi:10.1083/jcb.101.2.597 (1985).

217 Theriot, J. A. \& Mitchison, T. J. Actin microfilament dynamics in locomoting cells. Nature 352, 126-131 (1991).

218 Hu, K., Ji, L., Applegate, K. T., Danuser, G. \& Waterman-Storer, C. M. Differential transmission of actin motion within focal adhesions. Science 315, 111-115 (2007).

219 Eisenberg, E., Hill, T. L. \& Chen, Y. Cross-bridge model of muscle contraction. Quantitative analysis. Biophys. J. 29, 195227, doi:10.1016/S0006-3495(80)85126-5 (1980).

220 Chan, C. E. \& Odde, D. J. Traction dynamics of filopodia on compliant substrates. Science 322, 1687-1691 (2008).

221 Bangasser, B. L., Rosenfeld, S. S. \& Odde, D. J. Determinants of maximal force transmission in a motor-clutch model of cell traction in a compliant microenvironment. Biophys. J. 105, 581-592, doi:10.1016/j.bpj.2013.06.027 (2013).

222 Escribano, J., Sánchez, M. T. \& García-Aznar, J. M. A discrete approach for modeling cell-matrix adhesions. Computational Particle Mechanics 1, 117-130, doi:10.1007/s40571-014-0006-7 (2014).

223 Sabass, B. \& Schwarz, U. S. Modeling cytoskeletal flow over adhesion sites: competition between stochastic bond dynamics and intracellular relaxation. Journal of physics. Condensed matter : an Institute of Physics journal 22, 194112, doi:10.1088/0953-8984/22/19/194112 (2010).

224 Bangasser, B. L. \& Odde, D. J. Master equation-based analysis of a motor-clutch model for cell traction force. Cellular and molecular bioengineering 6, 449-459, doi:10.1007/s12195-013-0296-5 (2013).

225 Sens, P. Rigidity sensing by stochastic sliding friction. EPL 104, 38003 (2013).

226 Srinivasan, M. \& Walcott, S. Binding site models of friction due to the formation and rupture of bonds: state-function formalism, force-velocity relations, response to slip velocity transients, and slip stability. Physical review. E, Statistical, nonlinear, and soft matter physics 80, 046124, doi:10.1103/PhysRevE.80.046124 (2009).

\section{Competing interests}

The authors declare no competing interests.

\section{Acknowledgements}

We thank Hellyeh Hamidi for critical reading of the manuscript and text editing. This work was supported by the Spanish Ministry of Economy and Competitiveness (BFU2016-79916-P), the European Commission (H2020FETPROACT-01-2016-731957), the Generalitat de Catalunya (2017-SGR-1602), and Obra Social "La Caixa" (to P.R.C.), and ERC Consolidator grant 615258, and an Academy of Finland grant (to J.I.). IBEC is the recipient of a Severo Ochoa Award of Excellence from the MINECO. 


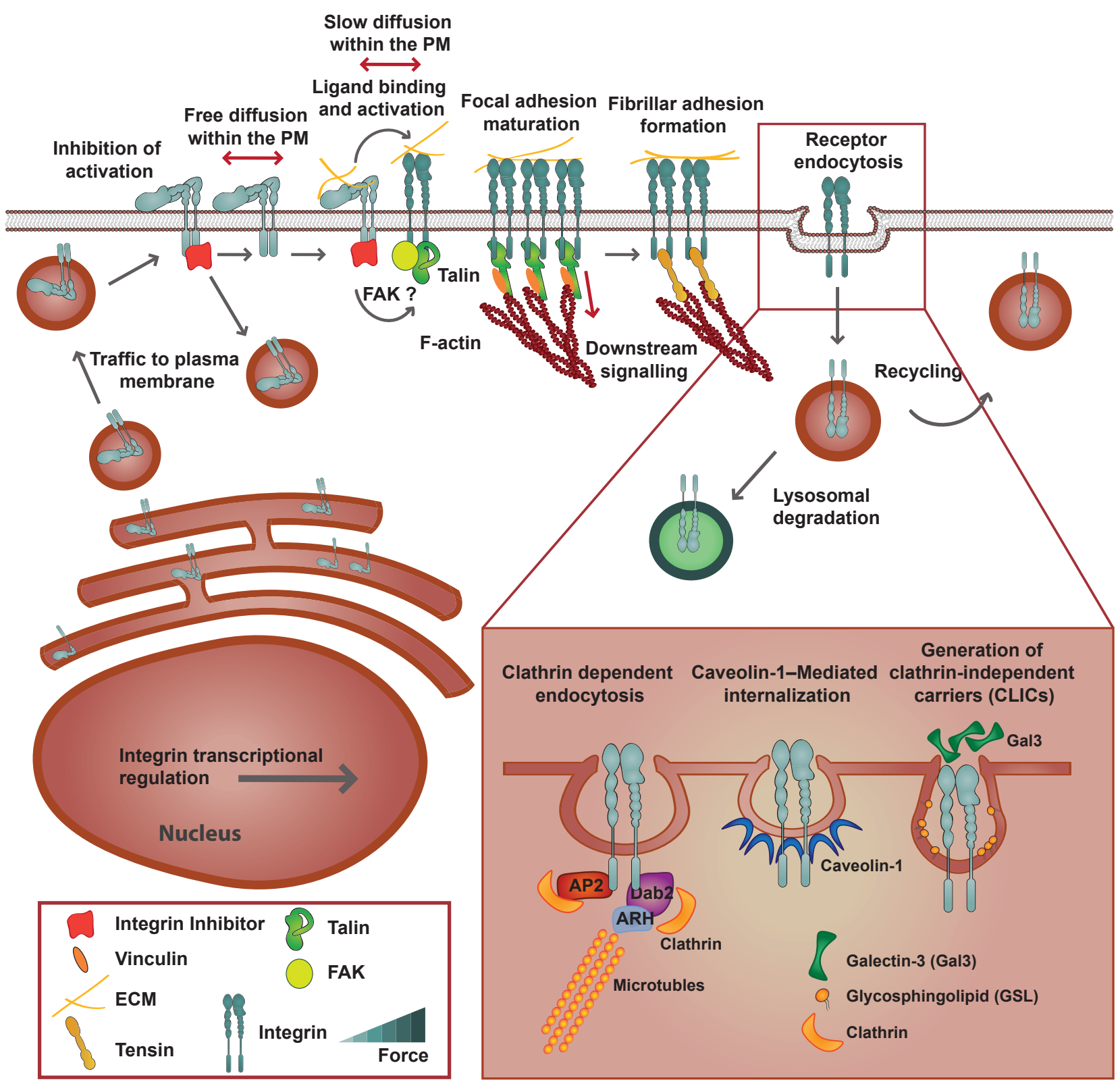

Figure 1 
a

$E C M$
$F<F_{B} \uparrow \quad F B \mid \uparrow / F>F_{B} \uparrow \uparrow$

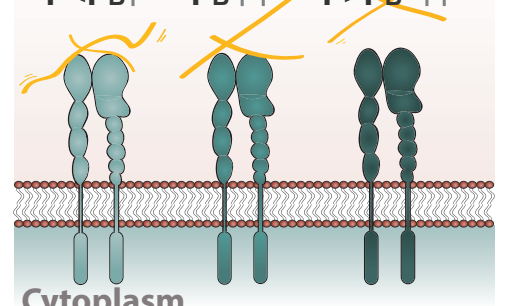

Cytoplasm

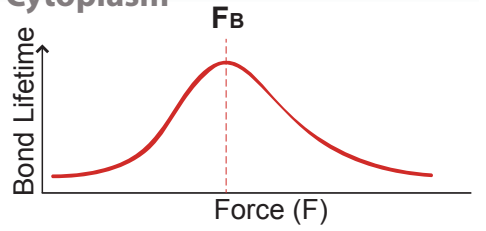

c Unfolding
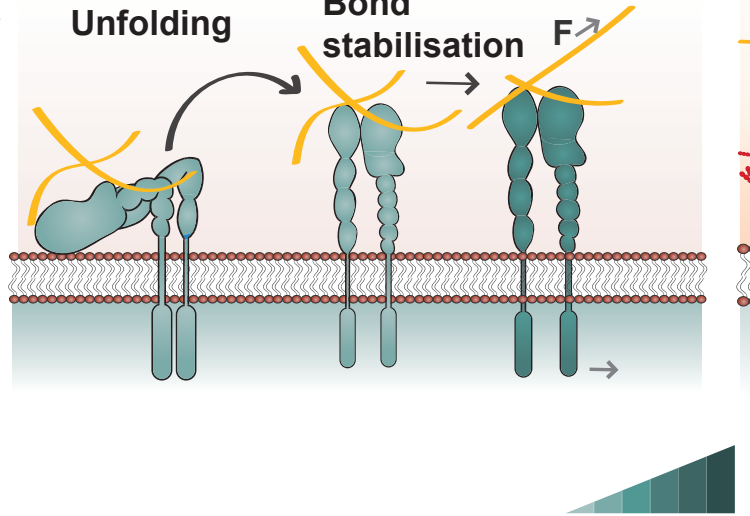

Force

b

d
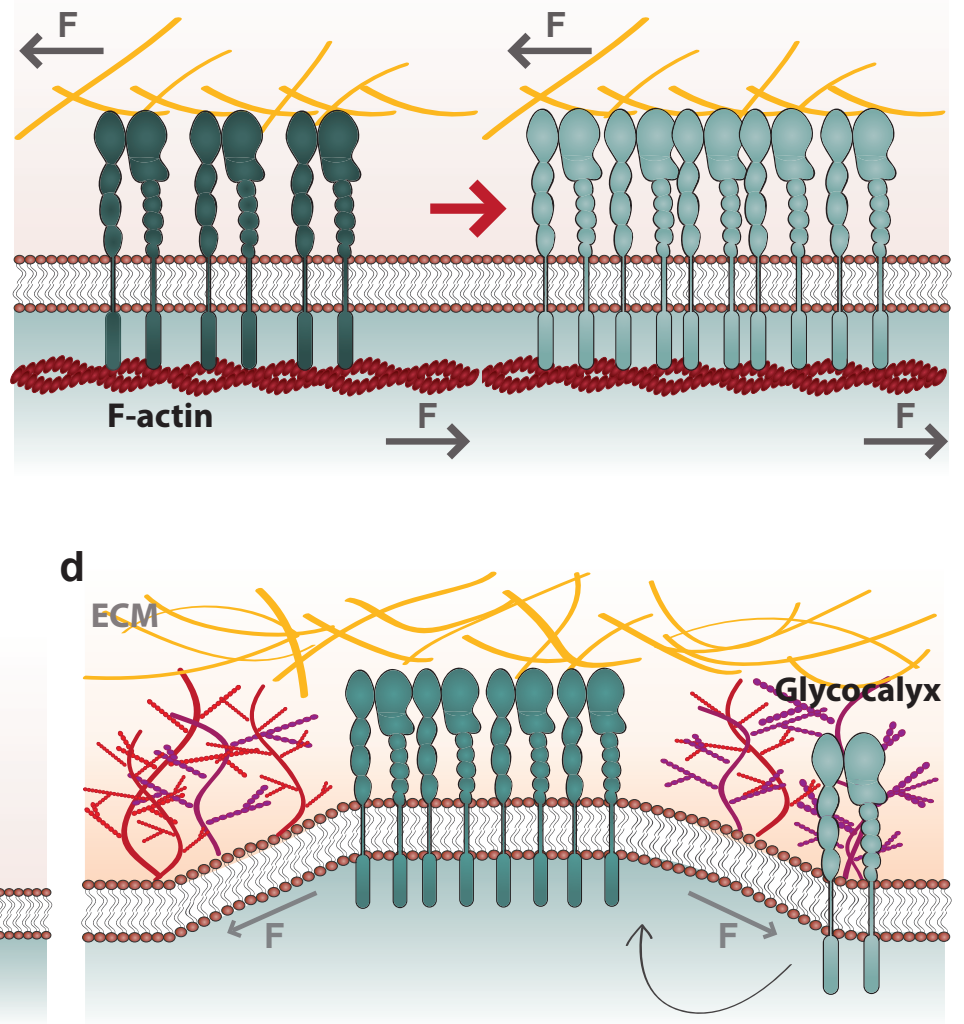
ECM 


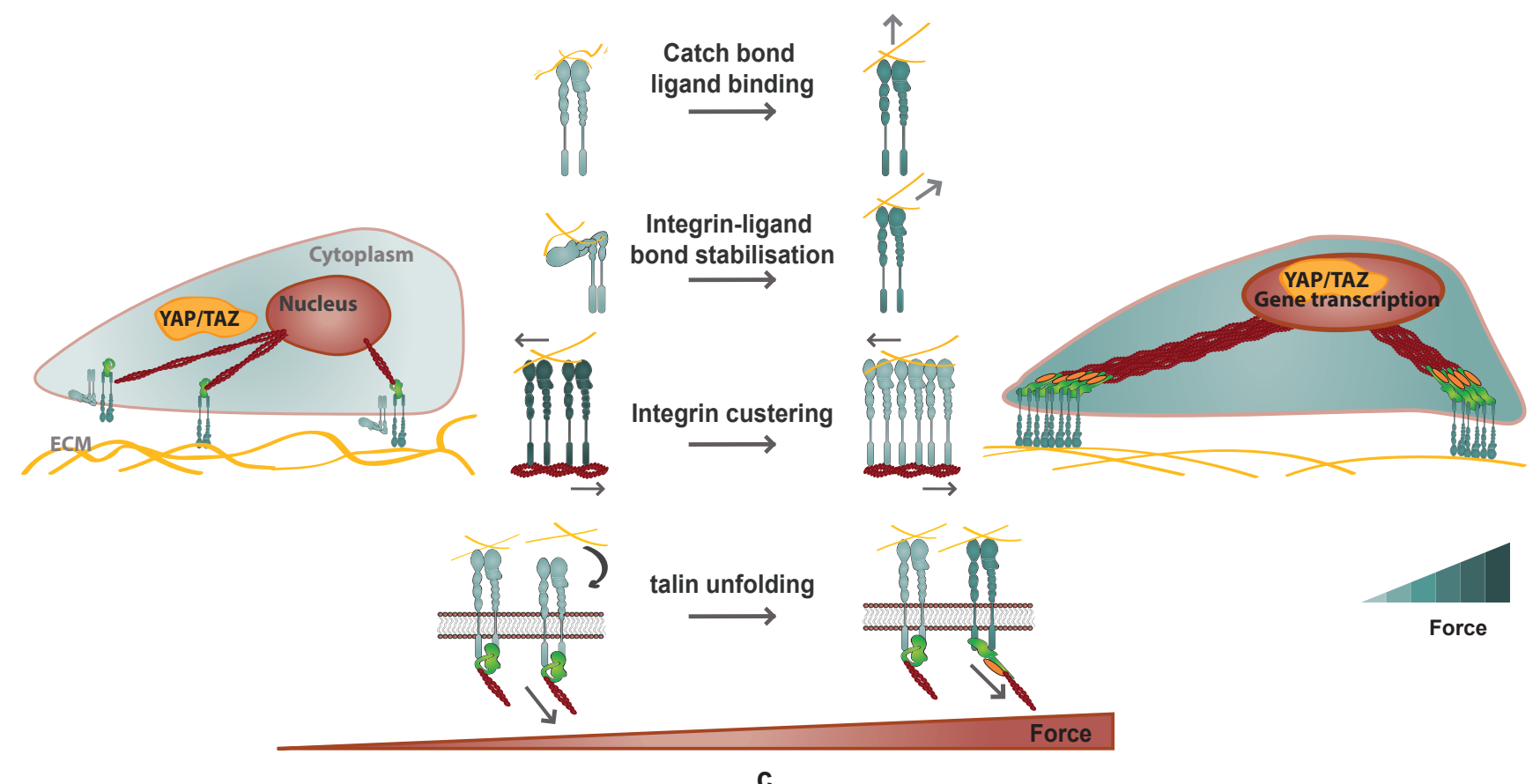

b

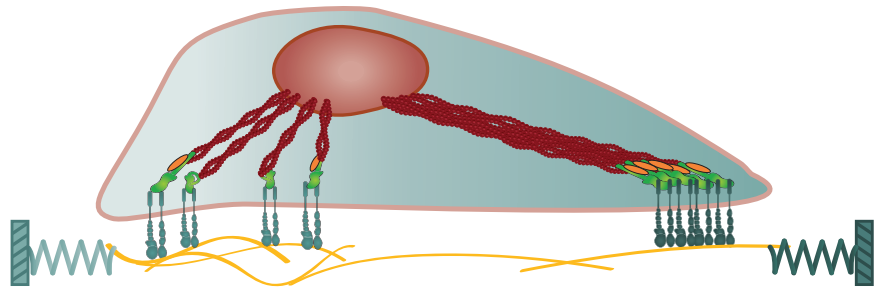

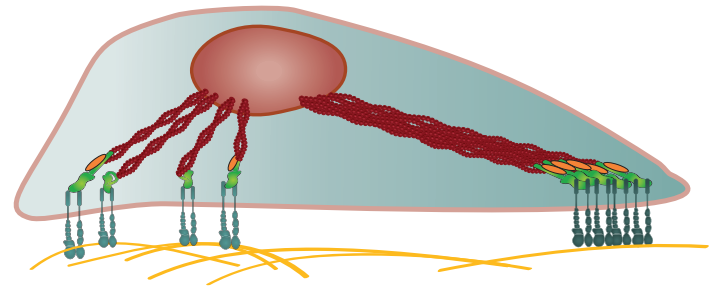




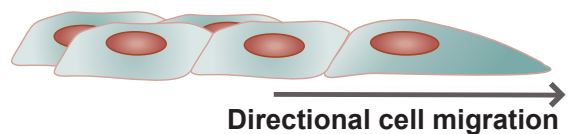

ECM stiffness

Durotaxis

Ligand density

Haptotaxis

Diffusible Ligand density

Chemotaxis

c

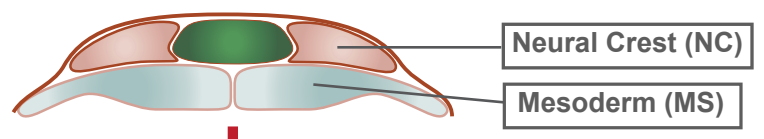

MS stiffening

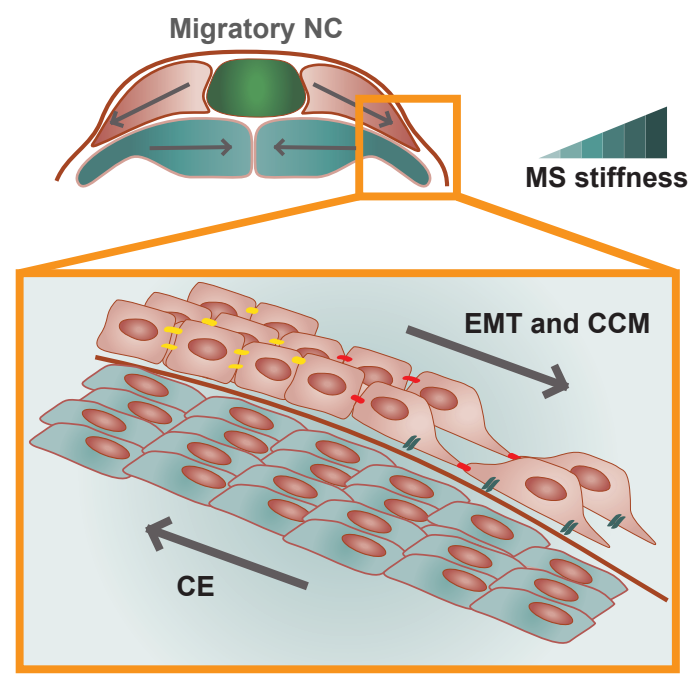

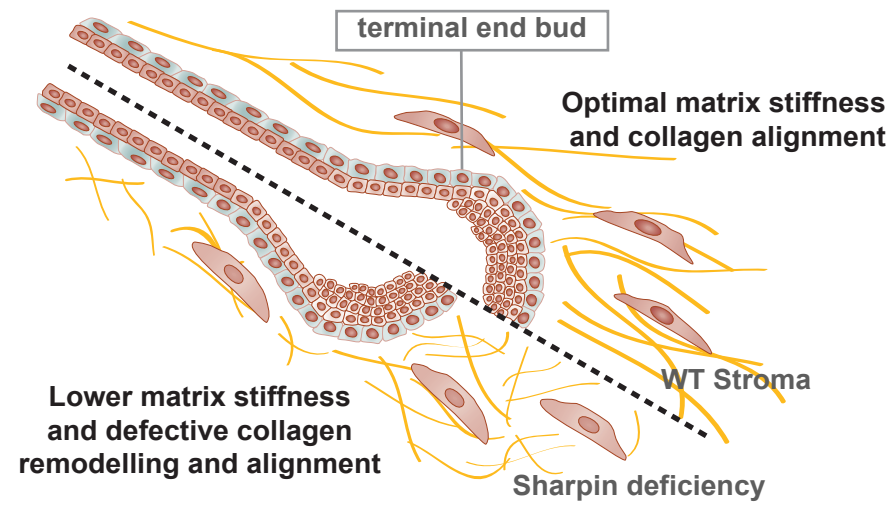

d
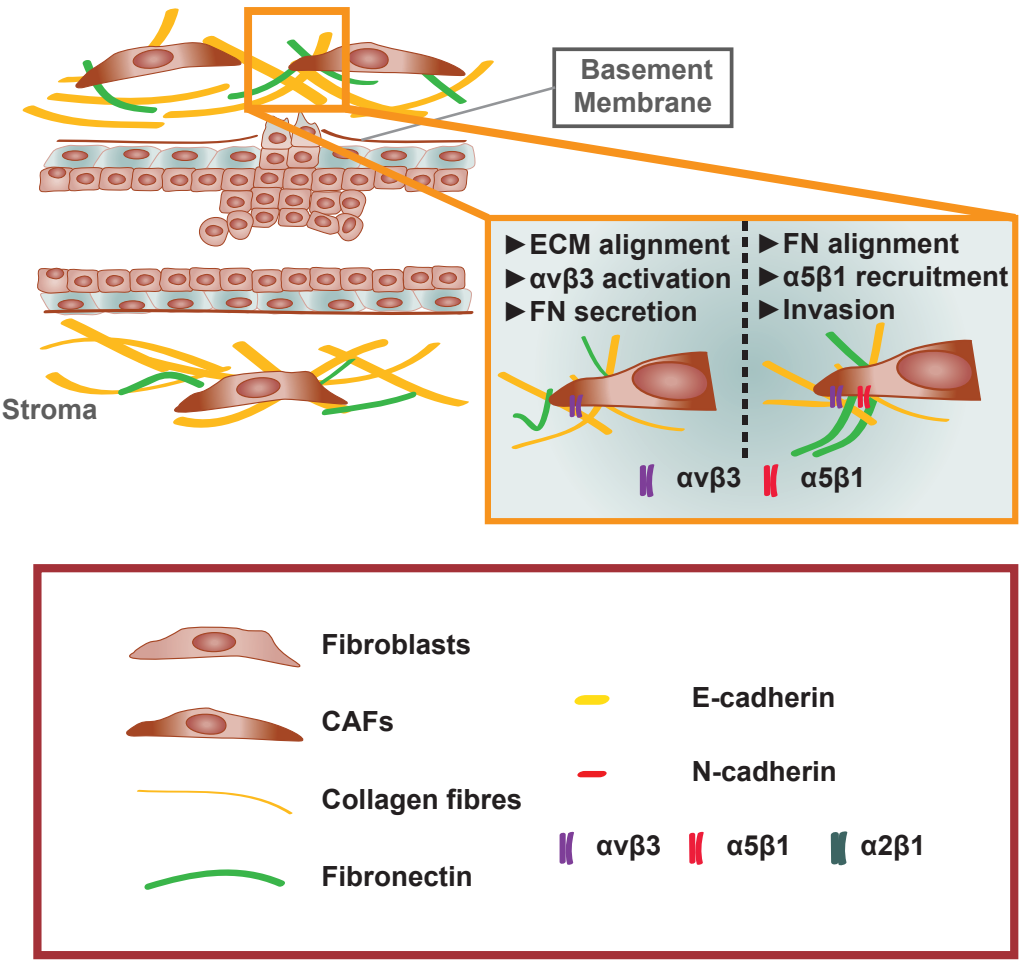


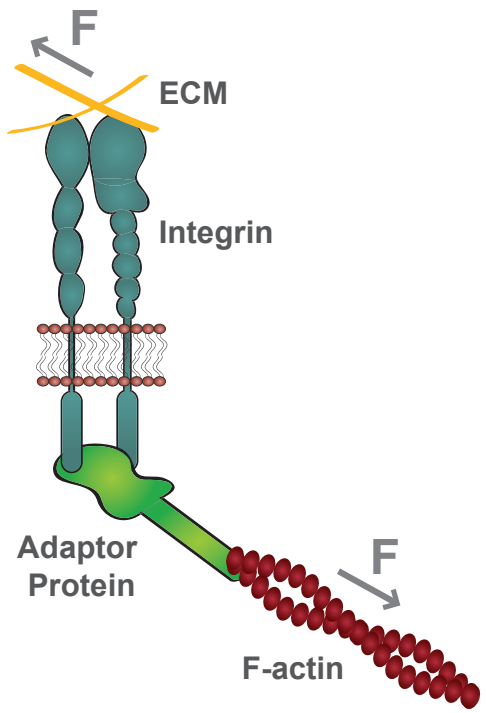

Box 1 